\title{
Graph-Theoretic Algorithms for Polynomial Optimization Problems
}

\author{
Somayeh Sojoudi, Ramtin Madani, Ghazal Fazelnia, and Javad Lavaei
}

\begin{abstract}
The objective of this tutorial paper is to study a general polynomial optimization problem using a semidefinite programming (SDP) relaxation. The first goal is to show how the underlying structure and sparsity of an optimization problem affect its computational complexity. Graph-theoretic algorithms are presented to address this problem based on the notions of low-rank optimization and matrix completion. By building on this result, it is then shown that every polynomial optimization problem admits a sparse representation whose SDP relaxation has a rank 1 or 2 solution. The implications of these results are discussed in details and their applications in decentralized control and power systems are also studied.
\end{abstract}

\section{INTRODUCTION}

Optimization theory deals with the minimization of an objective function subject to a set of constraints. This area plays a vital role in the design, control, operation, and analysis of real-world systems. The development of efficient optimization techniques and numerical algorithms has been an active area of research for many decades. The goal is to design a robust and scalable method that is able to find a global solution in polynomial time. This question has been fully answered for the class of convex optimization problems that includes all linear and some nonlinear problems [1]-[3]. Convex optimization has found a wide range of applications across engineering and economics [4]. In the past several years, a great effort has been devoted to casting realworld problems as convex optimization. Nevertheless, several classes of optimization problems, including polynomial optimization and quadratically constrained quadratic program (QCQP) as a special case, are nonlinear, non-convex, and NP-hard in the worst case [5], [6]. In particular, there is no known effective optimization technique for integer and combinatorial optimization as a small subclass of QCQP [7], [8]. Given a non-convex optimization, there are several techniques to find a solution that is locally optimal. However, seeking a global or near-global solution in polynomial time is a daunting challenge. There is a large body of literature on nonlinear optimization witnessing the complexity of this problem.

To reduce the computational complexity of a non-convex optimization, several convex relaxation methods based on linear matrix inequality (LMI), semidefinite programming

Somayeh Sojoudi is with the Langone Medical Center, New York University. Ramtin Madani, Ghazal Fazelnia, and Javad Lavaei are with the Electrical Engineering Department, Columbia University (Email: somayeh.sojoudi@nyumc.org, madani@ee.columbia.edu, gf2293@columbia.edu, and lavaei@ee. columbia.edu).

This work was supported by a Google Research Award, NSF CAREER Award and ONR YIP Award.
(SDP), and second-order cone programming (SOCP) have gained popularity [1], [9]. These techniques enlarge the possibly non-convex feasible set into a convex set characterizable via convex functions, and then provide the exact or a lower bound on the optimal objective value associated with a global solution. The SDP relaxation technique provides a lower bound on the minimum cost of the original problem, which can be used for various purposes such as the branch and bound algorithm [2]. To understand the quality of the SDP relaxation, its optimal objective value is shown to be at most $14 \%$ different from the optimal cost for the MAXCUT problem [10]. The maximum possible gap between the solution of a graph optimization and its SDP relaxation is defined as the Grothendieck constant of the graph [11], [12]. This constant has been derived for some special cases in [13]. The paper [14] shows how a complex SDP relaxation may solve the max-3-cut problem. This approach has been generalized in several papers [15]-[22]. If the SDP relaxation provides the same optimal objective value as the original problem, the relaxation is said to be exact. The exactness of the SDP relaxation has been verified for a variety of problems [23]-[26]. For instance, the work [27]-[30] has explored the SDP relaxation for the optimal power flow (OPF) problem, which is the most fundamental optimization problem for electrical power networks. That work shows that the relaxation is exact for a large class of OPF problems due to the physics of a power gird. The exactness of an SDP relaxation could be heavily formulation dependent. Indeed, a practical circuit optimization with four equivalent QCQPs is designed in [31], where only one of the formulations has an exact SDP relaxation.

In the case where the SDP relaxation is not exact, the existence of a low-rank SDP solution may still be helpful. To support this claim, a penalized SDP relaxation is proposed for the OPF problem in [31], [32] and successfully used to derive near-global solutions for 7000 instances of OPF (a near-global solution is a near-optimal solution that is close to a global solution with a known upper bound on its distance to global optimality). In a general context, the existence of a low-rank solution to matrix optimization problems with linear and LMI constraints has been extensively studied in the literature [33], [34]. The papers [35]-[37] provide an upper bound on the lowest rank among all solutions of a feasible LMI problem. Based on the same approach, a constructive method has been proposed in [38] to obtain a low-rank solution in polynomial time. Although the proven bound in [37] is tight in the worst case, many examples 
are known to possess solutions with a lower rank due to their underlying sparsity patterns [39], [40]. A rank-1 matrix decomposition technique is developed in [41] to find a rank1 solution whenever the number of constraints is small. This technique is extended in [42] to the complex SDP problem. The paper [43] presents a polynomial-time algorithm for finding an approximate low-rank solution.

This tutorial paper aims to study the SDP relaxation of a polynomial optimization through graph-theoretic notions. To this end, three problems will be addressed here. In Section II] the objective is to investigate how the structure of an optimization reduces the computational complexity. For this purpose, the structure of the optimization is mapped into a weighted graph and it is shown that the SDP relaxation is exact if the graph possesses certain properties. In Section III. it is shown that the SDP relaxation of a sparse optimization has a solution whose rank can be characterized in terms of the sparsity level of the problem. In Section IV, it is explained that every polynomial optimization admits a sparse representation whose SDP relaxation has a rank 1 or 2 matrix solution. In other words, it is shown that the NP hardness of polynomial optimization can be traced back to attaining a rank- 2 solution rather than a rank-1 solution. The techniques presented in this paper are illustrated on two notorious problems of "optimal distributed control" and "nonlinear power optimization" in Sections V and VI, respectively.

\section{A. Notations}

The notations used throughout this tutorial paper will be described below. $\mathbb{R}, \mathbb{C}, \mathbb{Z}_{+}, \mathbb{S}^{n}$, and $\mathbb{H}^{n}$ denote the sets of real numbers, complex numbers, nonnegative integer numbers, $n \times n$ symmetric matrices, and $n \times n$ Hermitian matrices, respectively. $\mathbb{S}_{+}^{n}$ and $\mathbb{H}_{+}^{n}$ denote the restrictions of $\mathbb{S}^{n}$ and $\mathbb{H}^{n}$ to positive semidefinite matrices. $\operatorname{Re}\{\mathbf{W}\}, \operatorname{Im}\{\mathbf{W}\}$, $\operatorname{rank}\{\mathbf{W}\}$, and trace $\{\mathbf{W}\}$ denote the real part, imaginary part, rank, and trace of a given scalar/matrix $\mathbf{W}$, respectively. The notation $\mathbf{W} \succeq 0$ means that $\mathbf{W}$ is Hermitian and positive semidefinite. Given a matrix $\mathbf{W}$, its $(l, m)$ entry is denoted as $W_{l m}$. The superscript $(\cdot)^{\text {opt }}$ is used to show the globally optimal value of an optimization parameter. The symbol $(\cdot)^{*}$ represents the conjugate transpose operator. $\measuredangle(x)$ represents the phase of a complex number $x$. The imaginary unit is denoted as "i", while " $i$ " is used for indexing. Given a set $\mathcal{T},|\mathcal{T}|$ denotes its cardinality. Given a graph $\mathcal{G},|\mathcal{G}|$ shows the number of its vertices. Given a number (vector) $\mathbf{x},|\mathbf{x}|$ denotes its absolute value (2-norm). Given an undirected graph $\mathcal{G}$, the notation $i \in \mathcal{G}$ means that $i$ is a vertex of $\mathcal{G}$. Moreover, the notation $(i, j) \in \mathcal{G}$ means that $(i, j)$ is an edge of $\mathcal{G}$ and besides $i<j$.

\section{Highly-Structured Optimization}

In this section, the objective is to investigate how the underlying sparsity or structure of an optimization problem affects its computational complexity. Our approach is to map the structure of the optimization into a weighted graph and relate the exactness of a conic relaxation for the optimization to certain properties of the graph.

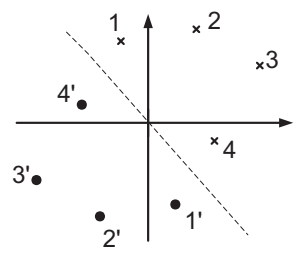

(a)

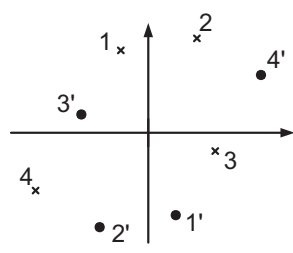

(b)
Fig. 1: In Figure (a), there exists a line separating x's (elements of $\mathcal{T}$ ) from o's (elements of $-\mathcal{T}$ ) so the set $\mathcal{T}$ is sign definite. In Figure (b), this is not the case.

\section{A. Definitions}

Before proceeding with this part, some definitions will be provided below.

Definition 1: A finite set $\mathcal{T} \subset \mathbb{R}$ is said to be sign definite with respect to $\mathbb{R}$ if its elements are either all negative or all nonnegative. $\mathcal{T}$ is called negative if its elements are negative and is called positive if its elements are nonnegative.

Definition 2: A finite set $\mathcal{T} \subset \mathbb{C}$ is said to be sign definite with respect to $\mathbb{C}$ if when the sets $\mathcal{T}$ and $-\mathcal{T}$ are mapped into two collections of points in $\mathbb{R}^{2}$, then there exists a line separating the two sets (note that any or all elements of the sets $\mathcal{T}$ and $-\mathcal{T}$ are allowed to lie on the separating line).

To illustrate Definition 2, consider a complex set $\mathcal{T}$ with four elements, whose corresponding points are labeled as 1 , 2, 3 and 4 in Figure 1 (a). The points corresponding to $-\mathcal{T}$ are labeled as 1', 2', 3' and 4' in the same picture. Since there exists a line separating x's (elements of $\mathcal{T}$ ) from o's (elements of $-\mathcal{T}$ ), the set $\mathcal{T}$ is sign definite. In contrast, if the elements of $\mathcal{T}$ are distributed according to Figure 1 (b), the set will no longer be sign definite. Note that Definition 2 is inspired by the fact that a real set $\mathcal{T}$ is sign definite with respect to $\mathbb{R}$ if $\mathcal{T}$ and $-\mathcal{T}$ are separable via a point (on the horizontal axis).

Definition 3: Given a graph $\mathcal{G}$, a cycle space is the set of all possible cycles in the graph. An arbitrary basis for this cycle space is called a "cycle basis".

Definition 4: In this work, a graph $\mathcal{G}$ is called weakly cyclic if every edge of the graph belongs to at most one cycle in $\mathcal{G}$ (i.e., the cycles of $\mathcal{G}$ are all edge-disjoint).

Definition 5: Consider a graph $\mathcal{G}$, a subgraph $\mathcal{G}_{s}$ of this graph and a matrix $\mathbf{W} \in \mathbb{H}^{|\mathcal{G}|}$. Define $\mathbf{W}\left\{\mathcal{G}_{s}\right\}$ as a submatrix of $\mathbf{W}$ located in the intersection of those rows and columns of $\mathbf{W}$ whose indices belong to the vertex set of $\mathcal{G}_{s}$. For instance, $\mathbf{W}\{(i, j)\}$ is obtained by intersecting rows $i, j$ with columns $i, j$ of $\mathbf{W}$, for every $(i, j) \in \mathcal{G}$.

\section{B. Problem Statement}

Consider an undirected graph $\mathcal{G}$ with $n$ vertices (nodes), where each edge $(i, j) \in \mathcal{G}$ has been assigned a nonzero edge weight set $\left\{c_{i j}^{(1)}, c_{i j}^{(2)}, \ldots, c_{i j}^{(k)}\right\}$ with $k$ real/complex numbers (note that the superscripts in the weights are not exponents). This graph is called a generalized weighted graph as every edge is associated with a set of weights as opposed to a single weight. Consider an unknown vector $\mathbf{x}=\left[\begin{array}{lll}x_{1} & \cdots & x_{n}\end{array}\right]$ 
belonging to $\mathbb{D}^{n}$, where $\mathbb{D}$ is either $\mathbb{R}$ or $\mathbb{C}$. For every $i \in \mathcal{G}$, $x_{i}$ is a variable associated with node $i$ of the graph $\mathcal{G}$. Define:

$$
\begin{aligned}
& \mathbf{y}=\left\{\left|x_{i}\right|^{2} \mid \forall i \in \mathcal{G}\right\}, \\
& \mathbf{z}=\left\{\operatorname{Re}\left\{c_{i j}^{(t)} x_{i} x_{j}^{*}\right\} \mid \forall(i, j) \in \mathcal{G}, t \in\{1, \ldots, k\}\right\}
\end{aligned}
$$

Note that $(i, j) \in \mathcal{G}$ means that $(i, j)$ is an edge of the graph and that $i<j$. The sets $\mathbf{y}$ and $\mathbf{z}$ can be regarded as two vectors, where

- $\mathbf{y}$ collects the quadratic terms $\left|x_{i}\right|^{2}$ 's (one term for each vertex).

- $\mathbf{z}$ collects the cross terms $\operatorname{Re}\left\{c_{i j}^{(t)} x_{i} x_{j}^{*}\right\}$ 's ( $k$ terms for each edge).

Although the above formulation deals with $\operatorname{Re}\left\{c_{i j}^{(t)} x_{i} x_{j}^{*}\right\}$ whenever $(i, j) \in \mathcal{G}$, it can handle terms of the form $\operatorname{Re}\left\{\alpha x_{j} x_{i}^{*}\right\}$ and $\operatorname{Im}\left\{\alpha x_{i} x_{j}^{*}\right\}$ for a complex weight $\alpha$. This can be carried out using the transformations:

$$
\begin{aligned}
& \operatorname{Re}\left\{\alpha x_{j} x_{i}^{*}\right\}=\operatorname{Re}\left\{\left(\alpha^{*}\right) x_{i} x_{j}^{*}\right\}, \\
& \operatorname{Im}\left\{\alpha x_{i} x_{j}^{*}\right\}=\operatorname{Re}\left\{(-\alpha \mathrm{i}) x_{i} x_{j}^{*}\right\}
\end{aligned}
$$

This part is concerned with the following optimization problem:

$$
\begin{aligned}
\min _{\mathbf{x} \in \mathbb{D}^{n}} & f_{0}(\mathbf{y}, \mathbf{z}) \\
\text { subject to } & f_{j}(\mathbf{y}, \mathbf{z}) \leq 0, \quad j=1,2, \ldots, m
\end{aligned}
$$

for given functions $f_{0}, \ldots, f_{m}$. The computational complexity of the above optimization problem depends in part on the structure of the functions $f_{j}$ 's. Regardless of these functions, the optimization problem (1) is intrinsically hard to solve (NP-hard in the worst case) because $\mathbf{y}$ and $\mathbf{z}$ are both nonlinear functions of $\mathbf{x}$. The objective is to convexify the second-order nonlinearity embedded in $\mathbf{y}$ and $\mathbf{z}$. To this end, notice that there exist two linear functions $l_{1}: \mathbb{C}^{n \times n} \rightarrow$ $\mathbb{R}^{n}$ and $l_{2}: \mathbb{C}^{n \times n} \rightarrow \mathbb{R}^{k \tau}$ such that $\mathbf{y}=l_{1}\left(\mathbf{x x}^{*}\right)$ and $\mathbf{z}=l_{2}\left(\mathbf{x x}^{*}\right)$, where $\tau$ denotes the number of edges in $\mathcal{G}$. Motivated by the above observation, if $\mathbf{x x}^{*}$ is replaced by a new matrix variable $\mathbf{W}$, then $\mathbf{y}$ and $\mathbf{z}$ both become linear in $\mathbf{W}$. This implies that the non-convexity induced by the quadratic terms $\operatorname{Re}\left\{c_{i j}^{(t)} x_{i} x_{j}\right\}$ 's and $\left|x_{i}\right|$ 's all disappear if the optimization problem (1) is reformulated in terms of $\mathbf{W}$. However, the optimal solution $\mathbf{W}$ may not be decomposable as $\mathbf{x x}^{*}$ unless some additional constraints are imposed on W. It is straightforward to verify that the optimization problem (1) is equivalent to

$$
\begin{array}{ll}
\min _{\mathbf{W}} & f_{0}\left(l_{1}(\mathbf{W}), l_{2}(\mathbf{W})\right) \\
\text { s.t. } & f_{j}\left(l_{1}(\mathbf{W}), l_{2}(\mathbf{W})\right) \leq 0, \quad j=1, \ldots, m \\
& \mathbf{W} \succeq 0, \\
& \operatorname{rank}\{\mathbf{W}\}=1
\end{array}
$$

where there is an implicit constraint that $\mathbf{W} \in \mathbb{S}^{n}$ if $\mathbb{D}=$ $\mathbb{R}$ and $\mathbf{W} \in \mathbb{H}^{n}$ if $\mathbb{D}=\mathbb{C}$. To reduce the computational complexity of the above problem, two actions can be taken: (i) removing the nonconvex constraint 2d, and (ii) relaxing the convex, but computationally-expensive, constraint $2 \mathrm{c}]$ to a set of simpler constraints on certain low-order submatrices of $\mathbf{W}$. Based on this methodology, three relaxations will be proposed for the optimization problem (1) next.

SDP relaxation: This optimization problem is defined as

$$
\begin{array}{ll}
\min _{\mathbf{W}} & f_{0}\left(l_{1}(\mathbf{W}), l_{2}(\mathbf{W})\right) \\
\text { s.t. } & f_{j}\left(l_{1}(\mathbf{W}), l_{2}(\mathbf{W})\right) \leq 0, \quad j=1, \ldots, m \\
& \mathbf{W} \succeq 0
\end{array}
$$

Reduced SDP relaxation: Choose a set of cycles $\mathcal{O}_{1}, \ldots, \mathcal{O}_{p}$ in the graph $\mathcal{G}$ such that they form a cycle basis. Let $\Omega$ denote the set of all subgraphs $\mathcal{O}_{1}, \ldots, \mathcal{O}_{p}$ as well as all edges of $\mathcal{G}$ that do not belong to any cycle in the graph (i.e., bridge edges). The reduced SDP relaxation is defined as

$$
\begin{aligned}
& \min _{\mathbf{W}} f_{0}\left(l_{1}(\mathbf{W}), l_{2}(\mathbf{W})\right) \\
& \text { s.t. } \quad f_{j}\left(l_{1}(\mathbf{W}), l_{2}(\mathbf{W})\right) \leq 0, \quad j=1, \ldots, m \\
& \mathbf{W}\left\{\mathcal{G}_{s}\right\} \succeq 0, \quad \forall \mathcal{G}_{s} \in \Omega
\end{aligned}
$$

SOCP relaxation: This optimization problem is defined as

$$
\begin{aligned}
& \min _{\mathbf{W}} f_{0}\left(l_{1}(\mathbf{W}), l_{2}(\mathbf{W})\right) \\
& \text { s.t. } f_{j}\left(l_{1}(\mathbf{W}), l_{2}(\mathbf{W})\right) \leq 0, \quad j=1, \ldots, m \\
& \mathbf{W}\{(i, j)\} \succeq 0, \quad \forall(i, j) \in \mathcal{G}
\end{aligned}
$$

The reason why the above optimization problem is called an SOCP problem is that the condition $\mathbf{W}\{(i, j)\} \succeq 0$ can be replaced by the linear and norm constraints

$$
\begin{aligned}
& \mathbf{W}_{i i}, \mathbf{W}_{j j} \geq 0 \\
& \mathbf{W}_{i i}+\mathbf{W}_{j j} \geq\left|\left[\begin{array}{lll}
\mathbf{W}_{i i} & \mathbf{W}_{j j} & \sqrt{2} \mathbf{W}_{i j}
\end{array}\right]\right|
\end{aligned}
$$

The above SDP, reduced SDP and SOCP relaxations target the non-convexity caused by the nonlinear relationship between $\mathbf{x}$ and $(\mathbf{y}, \mathbf{z})$. Note that these optimization problems are convex relaxations only when the functions $f_{0}, \ldots, f_{m}$ are convex. If any of these functions is nonconvex, additional relaxations might be needed to convexify the SDP, reduced SDP or SOCP optimization problem. Define $f^{\text {opt }}, f_{\mathrm{SDP}}^{\mathrm{opt}}, f_{\mathrm{r}-\mathrm{SDP}}^{\mathrm{opt}}$ and $f_{\mathrm{SOCP}}^{\mathrm{opt}}$ as the optimal solutions of the optimization problems (2), (3), (4) and (5), respectively. By comparing the feasible sets of these optimization problems, it can be concluded that

$$
f_{\mathrm{SOCP}}^{\mathrm{opt}} \leq f_{\mathrm{r}-\mathrm{SDP}}^{\mathrm{opt}} \leq f_{\mathrm{SDP}}^{\mathrm{opt}} \leq f^{\mathrm{opt}}
$$

Given a particular optimization problem of the form (1), if any of the above inequalities for $f^{\text {opt }}$ turns into an equality, the associated relaxation will be able to find the solution of the original optimization problem. In this case, it is said that the relaxation is "tight" or "exact". The objective of this part is to relate the exactness of the proposed relaxations to the topology of the graph $\mathcal{G}$ and its weight sets $\left\{c_{i j}^{(1)}, c_{i j}^{(2)}, \ldots, c_{i j}^{(k)}\right\}$ 's.

It is noteworthy that the aforementioned problem formulation can be easily generalized in two directions: 
- Allowance of weight sets with different cardinalities: The above problem formulation assumes that every edge weight set has $k$ elements. However, if the weight sets have different sizes, the trivial weight 0 can be added to each set multiple times in such a way that all expanded sets reach the same cardinality.

- Inclusion of linear terms in $\mathrm{x}$ : The optimization problem (1) is formulated in $\mathbf{x x}^{*}$ with no linear term in $\mathbf{x}$. This issue can be fixed by defining an expanded vector $\tilde{\mathbf{x}}$ as $\left[\begin{array}{cc}1 & \mathbf{x}^{*}\end{array}\right]^{*}$. Then, the matrix $\tilde{\mathbf{x}} \tilde{\mathbf{x}}^{*}$ needs to be replaced by a new matrix variable $\tilde{\mathbf{W}}$ under the constraint $\tilde{W}_{11}=1$.

\section{Exactness of Conic Relaxations}

Throughout this part, we assume that $f_{j}(\mathbf{y}, \mathbf{z})$ is monotonic in every entry of $\mathbf{z}$ for $j=0,1, \ldots, m$ (but possibly nonconvex in $\mathbf{y}$ and $\mathbf{z}$ ). With no loss of generality, suppose that $f_{j}(\mathbf{y}, \mathbf{z})$ is an increasing function with respect to all entries of $\mathbf{z}$.

The objective of this part is to study the interrelationship between $f_{\mathrm{SOCP}}^{\mathrm{opt}}, f_{\mathrm{r}-\mathrm{SDP}}^{\mathrm{opt}}, f_{\mathrm{SDP}}^{\mathrm{opt}}$ and $f^{\text {opt }}$. In particular, it is aimed to understand what properties the generalized weighted graph $\mathcal{G}$ should have to guarantee the exactness of some of the proposed relaxations. Some special cases of this problem have been studied in [44]-[46]. In what follows, various conditions will be provided to guarantee the exactness of the proposed SDP, reduced SDP and SOCP relaxation. The reader may refer to [39] for more details.

The following statements hold in both real and complex cases $\mathbb{D}=\mathbb{R}$ and $\mathbb{D}=\mathbb{C}$ :

i) The SDP relaxation is exact (i.e., $f_{\mathrm{SDP}}^{\mathrm{opt}}=f^{\mathrm{opt}}$ ) if and only if it has a rank-1 solution $\mathbf{W}^{\text {opt }}$.

ii) The reduced SDP relaxation is exact (i.e., $f_{\mathrm{r}-\mathrm{SDP}}^{\mathrm{opt}}=f^{\mathrm{opt}}$ ) if and only if it has a solution $\mathbf{W}^{\text {opt }}$ such that

$$
\operatorname{Rank}\left\{\mathbf{W}^{\text {opt }}\left\{\mathcal{G}_{s}\right\}\right\}=1, \quad \forall \mathcal{G}_{s} \in \Omega
$$

iii) The SOCP relaxation is exact (i.e., $f_{\text {SOCP }}^{\text {opt }}=f^{\text {opt }}$ ) if and only if it has a solution $\mathbf{W}^{\text {opt }}$ such that

$$
\operatorname{Rank}\left\{\mathbf{W}^{\text {opt }}\{(i, j)\}\right\}=1, \quad \forall(i, j) \in \mathcal{G}
$$

and that

$$
\sum \measuredangle \mathbf{W}_{i j}^{\mathrm{opt}}=0, \quad \forall r \in\{1,2, \ldots, p\}
$$

where the sum is taken over all directed edges $(i, j)$ of the oriented cycle $\overrightarrow{\mathcal{O}}_{r}$ (note that $\overrightarrow{\mathcal{O}}_{r}$ denotes a directed cycle corresponding to $\mathcal{O}_{r}$ ). Moreover, the same result holds even if the condition (8) is replaced by (7).

The above conditions reveal the role of the underlying graph of the optimization. By further simplifying these conditions, it can be shown that the SOCP, reduced SDP and SDP relaxations are all tight in the real-valued case $\mathbb{D}=\mathbb{R}$, provided each weight set $\left\{c_{i j}^{(1)}, c_{i j}^{(2)}, \ldots, c_{i j}^{(k)}\right\}$ is sign definite with respect to $\mathbb{R}$ and

$$
\prod_{(i, j) \in \mathcal{O}_{r}} \sigma_{i j}=(-1)^{\left|\mathcal{O}_{r}\right|}, \quad \forall r \in\{1, \ldots, p\}
$$

where $\sigma_{i j} \in\{-1,0,1\}$ shows the sign of the weight set associated with the edge $(i, j) \in \mathcal{G}$. This condition is naturally satisfied in three special cases:

- $\mathcal{G}$ is acyclic with arbitrary sign definite edge sets.

- $\mathcal{G}$ is bipartite with positive weight sets.

- $\mathcal{G}$ is arbitrary with negative weight sets.

If the SDP relaxation is not exact, it still has a low rank (rank-2) solution in two cases:

- $\mathcal{G}$ is acyclic (but with potentially indefinite weight sets).

- $\mathcal{G}$ is a weakly-cyclic bipartite graph with sign definite edge sets.

To study the complex-valued case $\mathbb{D}=\mathbb{C}$, assume that each edge set $\left\{c_{i j}^{(1)}, c_{i j}^{(2)}, \ldots, c_{i j}^{(k)}\right\}$ is sign definite with respect to $\mathbb{C}$. This assumption is trivially met if $k \leq 2$ or the weight set contains only real (or imaginary) numbers. It can be shown that:

- The SOCP, reduced SDP and SDP relaxations are all tight if $\mathcal{G}$ is acyclic.

- The SOCP, reduced SDP and SDP relaxations are tight if each weight set contains only real or imaginary numbers and

$$
\prod_{(i, j) \in \overrightarrow{\mathcal{O}}_{r}} \sigma_{i j}=(-1)^{\left|\mathcal{O}_{r}\right|}, \quad \forall r \in\{1, \ldots, p\}
$$

where $\sigma_{i j} \in\{0, \pm 1, \pm \mathrm{i}\}$ shows the sign of each weight set.

- The reduced SDP and SDP relaxations (but not necessarily the SOCP relaxation) are exact if $\mathcal{G}$ is bipartite and weakly cyclic with positive or negative real weight sets.

- The reduced SDP and SDP relaxations (but not necessarily the SOCP relaxation) are exact if $\mathcal{G}$ is a weakly cyclic graph with imaginary weight sets and the signs $\sigma_{i j}= \pm \mathrm{i}$.

Furthermore, if the graph $\mathcal{G}$ can be decomposed as a union of edge-disjoint subgraphs in an acyclic way in such a way that each subgraph has one of the above four structural properties, then the SDP relaxation is exact.

The above conditions will be examined on multiple examples below.

\section{Illustrative Examples}

Example 1: The minimization of an unconstrained bivariate quartic polynomial can be carried out via an SDP relaxation obtained from the first-order sum-of-squares technique [47]. In this example, we demonstrate how a computationally cheaper SOCP relaxation (in comparison to the foregoing SDP relaxation) can be used to solve the minimization of a structured bivariate quartic polynomial subject to an arbitrary number of structured bivariate quartic polynomials. To this end, we first consider the unconstrained case, where the goal is to minimize the polynomial

$$
f_{0}\left(x_{1}, x_{2}\right)=x_{1}^{4}+a x_{2}^{2}+b x_{1}^{2} x_{2}+c x_{1} x_{2}
$$

with the real-valued variables $x_{1}$ and $x_{2}$, for arbitrary coefficients $a, b, c \in \mathbb{R}$. In order to find the global minimum of 


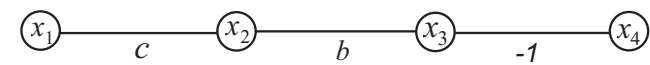

(a)

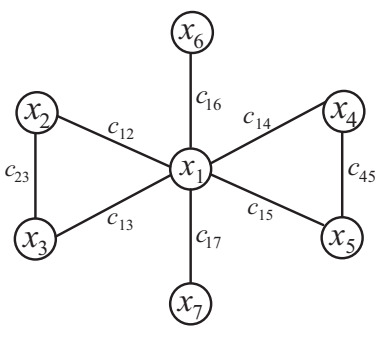

(b)

Fig. 2: Figures (a) and (b) show the weighted graph $\mathcal{G}$ for Examples 1 and 2, respectively.

this optimization problem, the standard convex optimization technique cannot readily be used due to the non-convexity of $f\left(x_{1}, x_{2}\right)$ in general. To address this issue, the above unconstrained minimization problem will be converted to a constrained quadratic optimization problem. More precisely, the problem of minimizing $f_{0}\left(x_{1}, x_{2}\right)$ can be reformulated in terms of $x_{1}, x_{2}$ and two auxiliary variables $x_{3}, x_{4}$ as:

$$
\begin{aligned}
\min _{\mathbf{x} \in \mathbb{R}^{4}} & x_{3}^{2}+a x_{2}^{2}+b x_{3} x_{2}+c x_{1} x_{2} \\
\text { s.t. } & x_{1}^{2}-x_{3} x_{4}=0, \quad x_{4}^{2}-1=0
\end{aligned}
$$

where $\mathbf{x}=\left[\begin{array}{llll}x_{1} & x_{2} & x_{3} & x_{4}\end{array}\right]^{*}$. The above optimization problem can be cast as follows:

$$
\begin{array}{cl}
\min _{\mathbf{x} \in \mathbb{R}^{4}, \mathbf{W} \in \mathbb{S}^{4}} & W_{33}+a W_{22}+b W_{23}+c W_{12} \\
\text { s.t. } & W_{11}-W_{34} \leq 0, \quad W_{44}-1=0
\end{array}
$$

and subject to the additional constraint $\mathbf{W}=\mathbf{x x}^{*}$. Note that $W_{11}-W_{34} \leq 0$ should have been $W_{11}-W_{34}=0$, but this modification does not change the solution. To eliminate the non-convexity induced by the constraint $\mathbf{W}=\mathrm{xx}^{*}$, one can use an SOCP relaxation obtained by replacing the constraint $\mathbf{W}=\mathbf{x x}^{*}$ with the convex constraints $\mathbf{W}\{(1,2)\} \succeq 0$, $\mathbf{W}\{(2,3)\} \succeq 0$ and $\mathbf{W}\{(3,4)\} \succeq 0$. To understand the exactness of this relaxation, the weighted graph $\mathcal{G}$ capturing the structure of the optimization problem (10) should be constructed. This graph is depicted in Figure 2 a). Since $\mathcal{G}$ is acyclic, the SOCP relaxation is exact for all values of $a, b, c$. Note that this does not imply that every solution $\mathbf{W}$ of the SOCP relaxation has rank 1. However, there is a simple systematic procedure for recovering a rank-1 solution from an arbitrary optimal solution of this relaxation.

Now, consider the constrained optimization case where a set of constraints

$f_{j}\left(x_{1}, x_{2}\right)=x_{1}^{4}+a_{j} x_{2}^{2}+b_{j} x_{1}^{2} x_{2}+c_{j} x_{1} x_{2} \leq d_{j} \quad j=1, \ldots, m$

is added to the optimization problem (9) for given coefficients $a_{j}, b_{j}, c_{j}, d_{j}$. In this case, the graph $\mathcal{G}$ depicted in Figure 2(a) needs to be modified by replacing its edge sets $\{b\}$ and $\{c\}$ with $\left\{b, b_{1}, \ldots, b_{m}\right\}$ and $\left\{c, c_{1}, \ldots, c_{m}\right\}$, respectively. The SOCP and SDP relaxations corresponding to the new optimization problem are exact as long as the sets $\left\{c, c_{1}, \ldots, c_{m}\right\}$ and $\left\{b, b_{1}, \ldots, b_{m}\right\}$ are both sign definite.

Example 2: Consider the optimization problem

$$
\min _{\mathbf{x} \in \mathbb{C}^{7}} \mathbf{x}^{*} M \mathbf{x} \quad \text { s.t. }\left|x_{i}\right|=1, \quad i=1,2, \ldots, 7
$$

where $M$ is a given Hermitian matrix. Assume that the weighted graph $\mathcal{G}$ depicted in Figure 2 (b) captures the structure of this optimization problem, meaning that (i) $M_{i j}=0$ for every pair $(i, j) \in\{1,2, \ldots 7\}$ such that $(i, j) \notin \mathcal{G}$, $(j, i) \notin \mathcal{G}$ and $i \neq j$, (ii) $M_{i j}$ is equal to the edge weight $c_{i j}$ for every $(i, j) \in \mathcal{G}$. The SDP relaxation of this optimization problem is as follows:

$$
\begin{aligned}
\min _{\mathbf{W} \in \mathbb{H}^{7}} & \operatorname{trace}\{M \mathbf{W}\} \\
\text { s.t. } & W_{11}=\cdots=W_{77}=1, \\
& \mathbf{W} \succeq 0
\end{aligned}
$$

Define $\mathcal{O}_{1}$ and $\mathcal{O}_{2}$ as the cycles induced by the vertex sets $\{1,2,3\}$ and $\{1,4,5\}$, respectively. Now, the reduced SDP and SOCP relaxations can be obtained by replacing the constraint $\mathbf{W} \succeq 0$ in the above optimization problem with certain small-sized constraints based on $\mathcal{O}_{1}$ and $\mathcal{O}_{2}$, as mentioned before. The following statements hold:

- The SDP, reduced SDP and SOCP relaxations are all exact in the case where $c_{12}, c_{13}, c_{14}, c_{15}, c_{23}, c_{45}$ are real numbers satisfying the inequalities $c_{12} c_{13} c_{23} \leq 0$ and $c_{14} c_{15} c_{45} \leq 0$.

- The SDP, reduced SDP and SOCP relaxations are all exact in the case where each of the sets $\left\{c_{12}, c_{13}, c_{23}\right\}$ and $\left\{c_{14}, c_{15}, c_{45}\right\}$ has at least one zero element.

- The SDP and reduced SDP are exact in the case where $c_{12}, c_{13}, c_{14}, c_{15}, c_{23}, c_{45}$ are imaginary numbers. Note that the SOCP relaxation may not be tight. To illustrate this fact, assume that the weights of the graph $\mathcal{G}$ are all equal to $+\mathrm{i}$ and that the diagonal entries of the matrix $M$ are zero. In this case, the SDP relaxation is known to be tight, but the optimal objective values of the SOCP and SDP relaxations are equal to two different numbers -16 and -14.3923 . Hence, the SOCP relaxation cannot be exact.

The above results demonstrate how the combined effect of the graph topology and the edge weights makes various relaxations exact for the quadratic optimization problem (12).

Example 3: Consider the optimization problem

$$
\min _{\mathbf{x} \in \mathbb{C}^{n}} \mathbf{x}^{*} M \mathbf{x} \quad \text { s.t. }\left|x_{j}\right|=1, \quad j=1,2, \ldots, m
$$

where $M$ is a symmetric real-valued matrix. It has been proven in [19] that this problem is NP-hard even in the case when $M$ is restricted to be positive semidefinite. Consider the graph $\mathcal{G}$ associated with the matrix $M$. The SDP and reduced SDP relaxations are exact for this optimization problem and therefore this problem is polynomial-time solvable with an arbitrary accuracy, provided that $\mathcal{G}$ is bipartite and weakly cyclic. To understand how well the SDP relaxation works, 
we pick $\mathcal{G}$ as a cycle with 4 vertices. Consider a randomly generated matrix $M$ :

$$
M=\left[\begin{array}{cccc}
0 & -0.0961 & 0 & -0.1245 \\
-0.0961 & 0 & -0.1370 & 0 \\
0 & -0.1370 & 0 & 0.7650 \\
-0.1245 & 0 & 0.7650 & 0
\end{array}\right]
$$

After solving the SDP relaxation numerically, an optimal solution $\mathbf{W}^{\text {opt }}$ is obtained as

$$
\mathbf{W}^{\text {opt }}=\left[\begin{array}{cccc}
1.0000 & 0.1767 & -0.5516 & 0.6505 \\
0.1767 & 1.0000 & 0.7235 & -0.6327 \\
-0.5516 & 0.7235 & 1.0000 & -0.9923 \\
0.6505 & -0.6327 & -0.9923 & 1.0000
\end{array}\right]
$$

This matrix has rank-2 and thus it seems as if the SDP relaxation is not exact. However, the fact is that this relaxation has a hidden rank-1 solution. To recover that solution, one can write $\mathbf{W}^{\text {opt }}$ as the sum of two rank-1 matrices, i.e., $\mathbf{W}^{\text {opt }}=\left(\mathbf{u}_{1}\right)\left(\mathbf{u}_{1}\right)^{*}+\left(\mathbf{u}_{2}\right)\left(\mathbf{u}_{2}\right)^{*}$ for two real vectors $\mathbf{u}_{1}$ and $\mathbf{u}_{1}$. It is straightforward to inspect that the complex-valued rank-1 matrix $\left(\mathbf{u}_{1}+\mathbf{u}_{2} \mathbf{i}\right)\left(\mathbf{u}_{1}+\mathbf{u}_{2} \mathbf{i}\right)^{*}$ is another solution of the SDP relaxation. Thus, $\mathbf{W}^{\mathrm{opt}}=\mathbf{u}_{1}+\mathbf{u}_{2} \mathrm{i}$ is an optimal solution of the optimization problem (13).

Example 4: Consider the optimization problem

$$
\begin{array}{ll}
\min _{\mathbf{x} \in \mathbb{C}^{n}} & \mathbf{x}^{*} M_{0} \mathbf{x} \\
\text { s.t. } & \mathbf{x}^{*} M_{j} \mathbf{x} \leq 0, \quad j=1,2, \ldots, m
\end{array}
$$

where $M_{0}, \ldots, M_{m}$ are symmetric real matrices, while $\mathbf{x}$ is an unknown complex vector. Similar to what was done in Example 1, a generalized weighted graph $\mathcal{G}$ can be constructed for this optimization problem. Regardless of the edge weights, as long as the graph $\mathcal{G}$ is acyclic, the SDP, reduced SDP and SOCP relaxations are all tight. As a result, this class of optimization problems is polynomialtime solvable with an arbitrary accuracy.

Example 5: As a generalization of linear programs, consider the non-convex optimization problem

$$
\begin{aligned}
\min _{\mathbf{x} \in \mathbb{R}^{n}} & \sum_{i=1}^{k} a_{0 i} e^{\mathbf{x}^{*} M_{0 i} \mathbf{x}}+\sum_{i=k+1}^{l} \mathbf{x}^{*} M_{0 i} \mathbf{x}+\mathbf{b}_{0}^{*} \mathbf{x} \\
\text { s.t. } & \sum_{i=1}^{k} a_{j i} e^{\mathbf{x}^{*} M_{j i} \mathbf{x}}+\sum_{i=k+1}^{l} \mathbf{x}^{*} M_{j i} \mathbf{x}+\mathbf{b}_{j}^{*} \mathbf{x} \leq 0
\end{aligned}
$$

for $j=1,2, \ldots, m$, where $a_{i j}$ 's are scalars, $\mathbf{b}_{j}$ 's are $n \times 1$ vectors, and $M_{i j}$ 's are $n \times n$ symmetric matrices. This problem involves linear terms, quadratic terms, and exponential terms with quadratic exponents. Using the technique stated in Section II-B the above optimization problem can be reformulated in terms of the rank-1 matrix $\tilde{\mathbf{x}} \tilde{\mathbf{x}}^{*}$ where $\tilde{\mathbf{x}}=$ $\left[\begin{array}{ll}1 & \mathbf{x}^{*}\end{array}\right]^{*}$, from which an SDP relaxation can subsequently be obtained by replacing the matrix $\tilde{\mathbf{x}} \tilde{\mathbf{x}}^{*}$ with a new matrix variable $\tilde{\mathbf{W}}$ under the constraint $\tilde{W}_{11}=1$. By mapping the structure of the optimization into a generalized weighted graph and noticing that $e^{x}$ is an increasing function in $x$, it can be concluded that the SDP relaxation is exact if the following conditions are all satisfied:
- $a_{j i}$ is nonnegative for every $j \in\{0, \ldots, m\}$ and $i \in$ $\{1, \ldots, k\}$.

- $\mathbf{b}_{j}$ is a non-positive vector for every $j \in\{0, \ldots, m\}$.

- $M_{j i}$ has only non-positive off-diagonal entries for every $j \in\{0, \ldots, m\}$ and $i \in\{1, \ldots, l\}$.

\section{Sparse Quadratic Optimization}

In the previous section, we explained that an optimization with two favorable structures may be solved through a conic relaxation: (i) sparsity and (ii) sign-definite coefficients. Although Condition (ii) is satisfied for certain problems (e.g., power optimization problems due to the passivity of a power grid), but it may be restrictive in general. In contrast, "sparsity" is a universal feature in real-world problems. The objective of this part is to understand how sparsity affects the computational complexity of a problem. More details on the results to be presented next can be found in [48].

\section{A. Low-Rank Positive Semidefinite Matrix Completion}

The low-rank positive semidefinite matrix completion problem aims to design the unknown entries of a partially filled matrix so that the resulting matrix becomes positive semidefinite with a minimum rank. This fundamental problem serves as a basis for studying the SDP relaxation for polynomial optimization problems. To introduce the problem, consider a simple graph $\mathcal{G}=(\mathcal{V}, \mathcal{E})$ with $n$ vertices together with a known matrix $\widehat{\mathbf{W}} \in \mathbb{S}_{+}^{n}(\mathcal{V}$ and $\mathcal{E}$ denote the vertex set and edge set of the graph). The goal is to solve the following optimization problem:

$$
\begin{array}{cll}
\min _{\mathbf{W} \in \mathbb{S}_{+}^{n}} & \operatorname{rank}\{\mathbf{W}\} & \\
\text { s.t. } & W_{i j}=\widehat{W}_{i j} & \forall(i, j) \in \mathcal{E} \\
& W_{k k}=\widehat{W}_{k k} & \forall k \in \mathcal{V}
\end{array}
$$

Note that the matrix $\mathbf{W}$ inherits the values of its diagonal and off-diagonal entries corresponding to the edges of $\mathcal{G}$ from the given matrix $\widehat{\mathbf{W}}$. Assume that the above optimization is feasible. This problem is difficult to tackle due to its nonconvex objective function. To reduce the complexity of the problem, we will propose two convex relaxations based on the graph notions of OS and treewidth.

Definition 6: Given a graph $\mathcal{G}=(\mathcal{V}, \mathcal{E})$, let $\mathcal{O}=\left\{o_{k}\right\}_{k=1}^{s}$ be a sequence of vertices of $\mathcal{G}$ with $s$ elements. Denote as $\mathcal{G}_{k}$ the subgraph induced by $\left\{o_{1}, \ldots, o_{k}\right\}$ for $k=1, \ldots, s$. Let $\mathcal{G}_{k}^{\prime}$ be the connected component of $\mathcal{G}_{k}$ containing $o_{k}$. $\mathcal{O}$ is called an OS-vertex sequence of $\mathcal{G}$ if, for every $k \in\{1, \ldots, s\}$, there exists a vertex $w_{k} \in \mathcal{V}$ with the following three properties:

1) $w_{k}$ is a neighbor of $o_{k}$, i.e., $\left(o_{k}, w_{k}\right) \in \mathcal{E}$

2) $w_{k}$ does not belong to the set $\left\{o_{1}, o_{2}, \ldots, o_{k}\right\}$

3) $w_{k}$ is not connected to any vertex in $\mathcal{G}_{k}^{\prime}$ other than $o_{k}$ Denote the maximum cardinality among all OS-vertex sequences of $\mathcal{G}$ as $\operatorname{OS}(\mathcal{G})$ [49] .

Figure 3 shows the construction of a maximal OS-vertex sequence of the Petersen graph. Dashed lines and bold lines highlight nonadjacency and adjacency, respectively, to show how $w_{k}$ at each step satisfies the conditions of Definition 6 

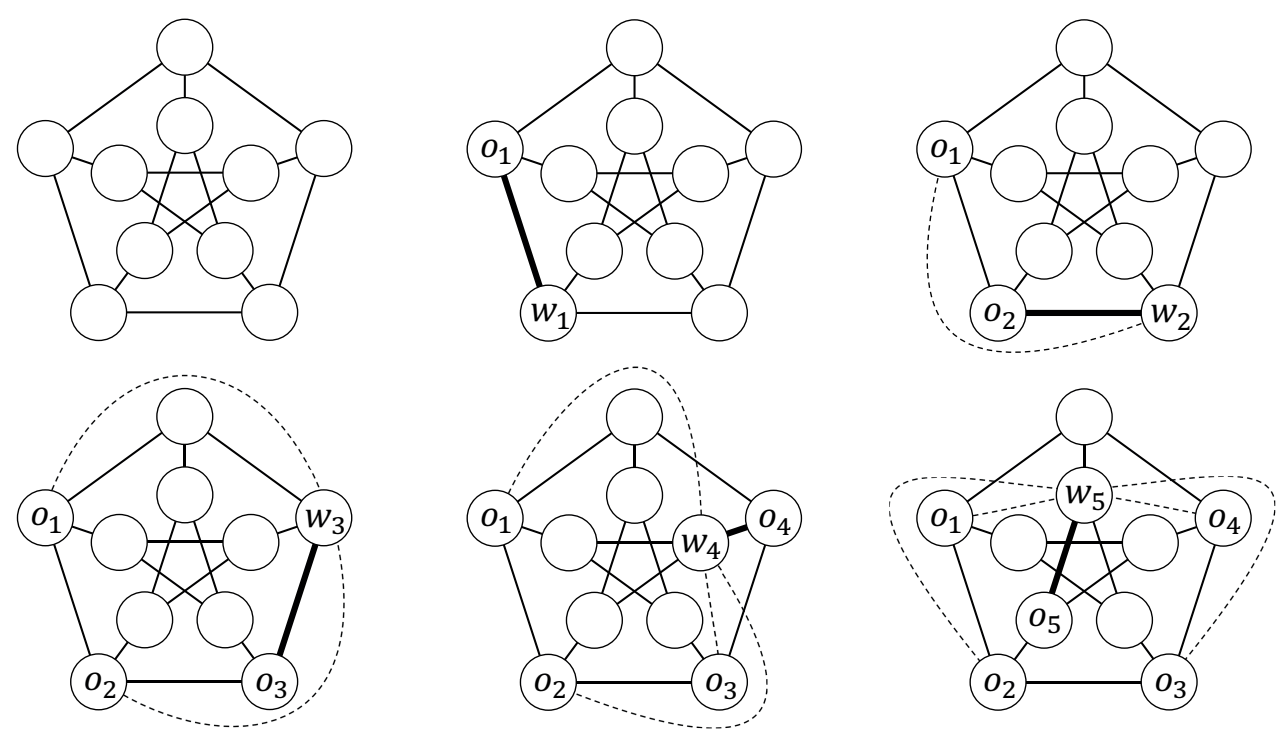

Fig. 3: A maximal OS-vertex sequence for the Petersen graph
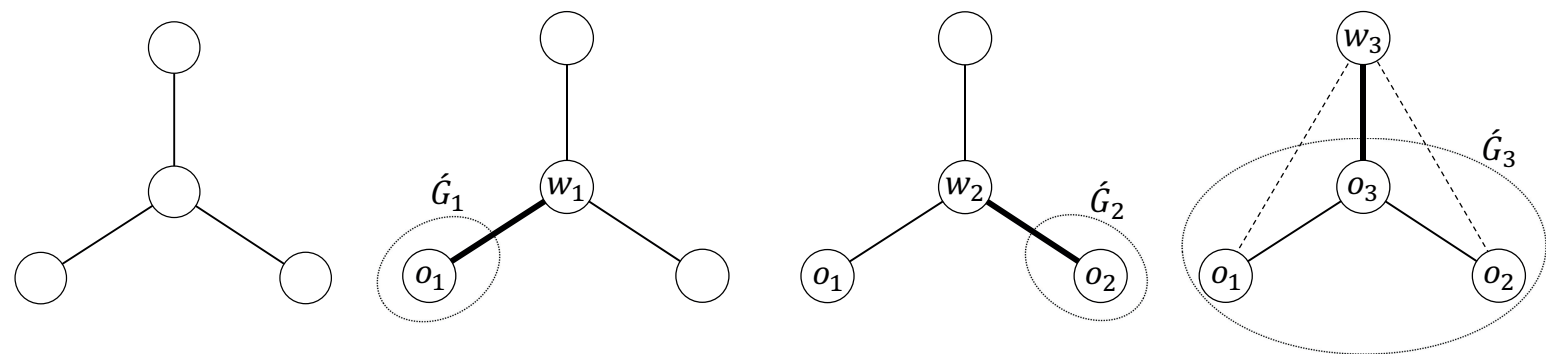

Fig. 4: A maximal OS-vertex sequence for a tree

Figure 4 illustrates the procedure of finding a maximal OSvertex sequence for a tree. The connected component of each $o_{k}$ in the subgraph induced by $\left\{o_{1}, \ldots, o_{k}\right\}$ is also shown. Notice that although $w_{2}$ is connected to $o_{1}$, it is a valid choice because $o_{1}$ and $o_{2}$ do not share the same connected component in $G_{2}$. To develop a convex relaxation for the matrix completion problem (14), let $\mathcal{G}^{c}=\left(\mathcal{V}^{c}, \mathcal{E}^{c}\right)$ denote an arbitrary graph such that $\mathcal{V}^{c}=\mathcal{V}$ and $\mathcal{E}^{c} \cap \mathcal{E}=\phi$.

Convex Relaxation I: This problem is defined as

$$
\begin{array}{cll}
\min _{\mathbf{W} \in \mathbb{S}_{+}^{n}} & \sum_{(i, j) \in \mathcal{E}^{c}} t_{i j} W_{i j} & \\
\text { s.t. } & W_{i j}=\widehat{W}_{i j} & \forall(i, j) \in \mathcal{E} \\
& W_{k k}=\widehat{W}_{k k} & \forall k \in \mathcal{V}
\end{array}
$$

where $t_{i j}$ 's are arbitrary nonzero scalars.

Assume that the above problem has a strictly feasible (positive definite) point $\mathbf{W}$ (see [48] for a discussion on the removal of this mild assumption). Then, every solution of Convex Relaxation I, denoted as $\mathbf{W}^{\text {opt }}$, satisfies the inequality

$$
\operatorname{rank}\left\{\mathbf{W}^{\mathrm{opt}}\right\} \leq n-\min _{\mathcal{G}^{s}}\left\{\operatorname{OS}\left(\mathcal{G}^{s} \cup \mathcal{G}^{c}\right) \mid \mathcal{G}^{s} \subseteq \mathcal{G}\right\}
$$

where
- The notation $\mathcal{G}^{s} \subseteq \mathcal{G}$ means that $\mathcal{G}^{s}$ is a graph with $n$ vertices whose edge set is a subset of the edge set of $\mathcal{G}$.

- $\mathcal{G}^{s} \cup \mathcal{G}^{c}$ denotes the edge-wise union of the graphs $\mathcal{G}^{s}$ and $\mathcal{G}^{c}$.

Note that the inequality (16) holds for all possible nonzero values of the coefficients $t_{i j}$ 's. Hence, the convex optimization (15) provides a suboptimal solution for the non-convex problem (14) together with an upper bound on its optimal objective value. Roughly speaking, a suitable choice of $\mathcal{G}^{c}$ makes the upper bound $n-\min _{\mathcal{G}^{s} \subseteq \mathcal{G}} \operatorname{OS}\left(\mathcal{G}^{s} \cup \mathcal{G}^{c}\right)$ very small for a large class of spare graphs $\mathcal{G}$ 's. To elaborate on this statement, we render the notion of tree decomposition.

Definition 7: Given a graph $\mathcal{G}=(\mathcal{V}, \mathcal{E})$, a tree $\mathcal{T}$ is called a tree decomposition of $\mathcal{G}$ if it satisfies the following properties:

1) Every node of $\mathcal{T}$ corresponds to and is identified by a subset of $\mathcal{V}$. Alternatively, each node of $\mathcal{T}$ is regarded as a group of vertices of $\mathcal{G}$.

2) Every vertex of $\mathcal{G}$ is a member of at least one node of $\mathcal{T}$.

3) $\mathcal{T}_{k}$ is a connected graph for $k=1,2, \ldots, n$, where $\mathcal{T}_{k}$ denotes the subgraph of $\mathcal{T}$ induced by all nodes of $\mathcal{T}$ containing the vertex $k$ of $\mathcal{G}$. 

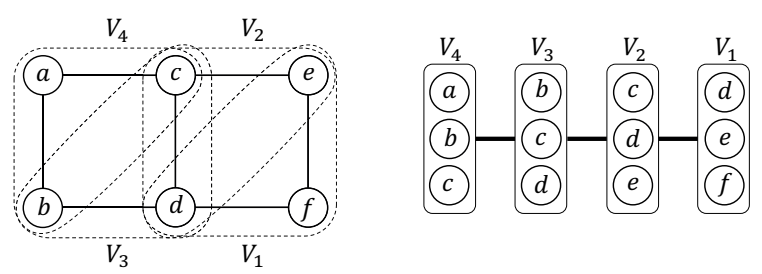

Fig. 5: A minimal tree decomposition for a ladder

4) The subtrees $\mathcal{T}_{i}$ and $\mathcal{T}_{j}$ have a node in common for every $(i, j) \in \mathcal{E}$.

The width of a tree decomposition is the cardinality of its biggest node minus one (recall that each node of $\mathcal{T}$ is indeed a set containing a number of vertices of $\mathcal{G}$ ). The treewidth of $\mathcal{G}$ is the minimum width over all possible tree decompositions of $\mathcal{G}$ and is denoted by $\operatorname{tw}(\mathcal{G})$.

Note that the treewidth of a tree is equal to 1 . Figure 5 shows a graph $\mathcal{G}$ with 6 vertices named $a, b, c, d, e, f$, together with its minimal tree decomposition $\mathcal{T}$. Every node of $\mathcal{T}$ is a set containing three members of $\mathcal{V}$. The width of this decomposition is therefore equal to 2 . A large class of real-world graphs are believed to have small treewidth numbers. As an example, we will verify the treewidth of $\mathcal{G}$ for two different applications later in this paper.

Given a tree decomposition $\mathcal{T}$ of the graph $\mathcal{G}$ with width $t$, with no loss of generality assume that all nodes of $\mathcal{T}$ have the same cardinality (see [48] for the general case). We design a graph $\mathcal{G}^{c}$ according to the following procedure:

- Step 1: Initialize $\mathcal{T}^{c}$ as $\mathcal{T}$ and $\mathcal{G}^{c}$ as a graph with $n$ vertices and no edges.

- Step 2: Identify a node of $\mathcal{T}^{c}$ with degree 1 (i.e., a leaf). Let $V_{1}$ and $V_{2}$ denote this node and its unique neighbor in $\mathcal{T}^{c}$ (note that $V_{1}, V_{2} \in \mathcal{V}$ because each node of $\mathcal{T}^{c}$ is indeed a collection of vertices of $\mathcal{G}$ )

- Step 3: Let $\left\{p_{1}, \ldots, p_{g}\right\}$ and $\left\{q_{1}, \ldots, q_{g}\right\}$ represent the sets $V_{1}-V_{2}$ and $V_{2}-V_{1}$, respectively. Add the edges $\left(p_{1}, q_{1}\right), \ldots,\left(p_{g}, q_{g}\right)$ to $\mathcal{G}^{c}$ and then remove node $V_{1}$ from $\mathcal{T}^{c}$.

- Step 4: Jump to Step 2 if $\mathcal{T}^{c}$ is still nonempty.

It can be shown that

$$
n-\min _{\mathcal{G}^{s}}\left\{\operatorname{OS}\left(\mathcal{G}^{s} \cup \mathcal{G}^{c}\right) \mid \mathcal{G}^{s} \subseteq \mathcal{G}\right\} \leq t+1
$$

for this choice of $\mathcal{G}^{c}$. Hence, the convex Optimization $(15)$ is able to provide a suboptimal solution for Optimization (14) with the property that $\operatorname{rank}\left\{\mathbf{W}^{\text {opt }}\right\} \leq t+1$. In particular, if an optimal tree decomposition is deployed for the construction of $\mathcal{G}^{c}$, then $\operatorname{rank}\left\{\mathbf{W}^{\text {opt }}\right\} \leq \operatorname{tw}(\mathcal{G})+1$ for all nonzero values of the coefficients $t_{i j}$ 's. Note that the existence of a solution for Optimization (14) of rank at most tw $(\mathcal{G})+1$ has already been proved in [50] for real-valued problems, but the technique stated above works for both real and complex problems. In addition, the above technique designs infinitely many optimization problems, each of which returns such a solution. The importance of this result will become clear later in the paper. The problem of finding a tree decomposition of minimum width is NP-complete in general [51]. Nevertheless, for a fixed integer $t$, the problem of checking the existence of a tree decomposition of width $t$ and finding such a decomposition (if any) can be solved in linear time [52], [53]. It is interesting to note that the treewidth for the optimal decentralized control problem (to be stated later in the paper) is equal to 2 due to its extreme sparsity.

Assume that $\mathcal{G}$ is a large-scale graph with no clear sparsity pattern. In this case, it may be very difficult to find a good tree decomposition or directly design a subgraph $\mathcal{G}^{c}$ minimizing the upper bound $n-\min _{\mathcal{G}^{s} \subseteq \mathcal{G}} \operatorname{OS}\left(\mathcal{G}^{s} \cup \mathcal{G}^{c}\right)$. Under this circumstance, we introduce another convex relaxation for Optimization (14).

Convex Relaxation II: This optimization problem is defined as

$$
\begin{array}{cll}
\min _{\mathbf{W} \in \mathbb{H}_{+}^{n}} & \sum_{(i, j) \in \mathcal{E} \cup \mathcal{E}^{c}} t_{i j} \operatorname{Im}\left\{W_{i j}\right\} & \\
\text { s.t. } & \operatorname{Re}\left\{W_{i j}\right\}=\widehat{W}_{i j} & \forall(i, j) \in \mathcal{E} \\
& W_{k k}=\widehat{W}_{k k} & \forall k \in \mathcal{V}
\end{array}
$$

with nonzero coefficients $t_{i j}$ 's, where the variable of the optimization is a complex-valued matrix. Assume that the above problem has a strictly feasible (positive definite) point $\mathbf{W}$. Let $\mathbf{W}^{\text {opt }}$ denote an arbitrary solution of this optimization. The matrix $\operatorname{Re}\left\{\mathbf{W}^{\text {opt }}\right\}$ turns out to be a suboptimal solution of the matrix completion problem (14) satisfying the inequality

$$
\operatorname{rank}\left\{\operatorname{Re}\left\{\mathbf{W}^{\text {opt }}\right\}\right\} \leq 2\left(n-\mathrm{OS}\left(\mathcal{G} \cup \mathcal{G}^{c}\right)\right)
$$

At the cost of adding the factor 2, the bound provided in (19) is simpler than the one given in (16) due to obviating the need to take the minimum of $\mathrm{OS}(\cdot)$ over a set of subgraphs $\mathcal{G}^{s}$ 's. The above bound is quite useful because it is a small number for a large class of sparse graphs, even in the case where $\mathcal{G}^{c}$ is considered as a trivial graph with no edges. This bound reduces to 4 for the optimal distributed control problem to be studied later in the paper.

The bounds provided in (16) and (19) can both be improved (for non-chordal graphs) by supplanting OS(.) with $\operatorname{msr}(\cdot)$, where "msr" stands for the minimum semidefinite rank of a graph [54]-[56]. Indeed, $\operatorname{msr}(\mathcal{G})$ is equal to the smallest rank of all positive semidefinite matrices with the same support as the adjacency matrix of $\mathcal{G}$.

\section{B. Sparse Quadratic Optimization}

Consider the standard non-convex quadraticallyconstrained quadratic program $(\mathrm{QCQP})$ :

$$
\begin{aligned}
& \min _{\mathbf{x} \in \mathbb{R}^{n-1}} \quad \mathbf{x}^{*} A_{0} \mathbf{x}+2 \mathbf{b}_{0}^{*} \mathbf{x}+c_{0} \\
& \text { s.t. } \quad \mathbf{x}^{*} A_{k} \mathbf{x}+2 \mathbf{b}_{k}^{*} \mathbf{x}+c_{k} \leq 0 \quad \text { for } \quad k=1, \ldots, m
\end{aligned}
$$

where $A_{k} \in \mathbb{R}^{(n-1) \times(n-1)}, \mathbf{b}_{k} \in \mathbb{R}^{n-1}$ and $c_{k} \in \mathbb{R}$, for $k=0, \ldots, m$. Define

$$
M_{k} \triangleq\left[\begin{array}{cc}
c_{k} & \mathbf{b}_{k}^{*} \\
\mathbf{b}_{k} & A_{k}
\end{array}\right]
$$


Each function $f_{k}$ has the linear representation $f_{k}(x)=$ trace $\left\{M_{k} \mathbf{W}\right\}$ where

$$
\mathbf{W} \triangleq\left[\begin{array}{ll}
1 & x^{*}
\end{array}\right]^{*}\left[\begin{array}{ll}
1 & x^{*}
\end{array}\right]
$$

Conversely, an arbitrary matrix $\mathbf{W} \in \mathbb{S}^{n}$ can be factorized as (22) if it satisfies three properties: $W_{11}=1, \mathbf{W} \succeq 0$, and $\operatorname{rank}\{\mathbf{W}\}=1$. Therefore, optimization 20) can be reformulated as follows:

$$
\begin{array}{cl}
\min _{\mathbf{W} \in \mathbb{S}^{n}} & \operatorname{trace}\left\{M_{0} \mathbf{W}\right\} \\
\text { s.t. } & \operatorname{trace}\left\{M_{k} \mathbf{W}\right\} \leq 0 \quad \text { for } \quad k=1, \ldots, m \\
& W_{11}=1 \\
& \mathbf{W} \succeq 0 \\
& \operatorname{rank}\{\mathbf{W}\}=1
\end{array}
$$

In the above reformulation of QCQP, the constraint $\operatorname{rank}\{\mathbf{W}\}=1$ carries all the non-convexity. Neglecting this constraint yields an SDP relaxation [57], [58]. The existence of a rank-1 solution for this SDP relaxation guarantees the equivalence between the original QCQP and its relaxed problem. Let $\widehat{\mathbf{W}}$ denote an arbitrary solution of the SDP relaxation of optimization (20). It is straightforward to verify that $\widehat{\mathbf{W}}$ may become full rank and yet there would exist a low-rank solution. Indeed, it can naturally occur that the SDP relaxation will have infinitely many solutions and therefore a solution with the lowest rank should be sought.

Low-Rank Solution: In an effort to find a low-rank SDP solution, let $\mathcal{G}=(\mathcal{V}, \mathcal{E})$ be a graph with $n$ vertices such that $(i, j) \in \mathcal{G}$ if and only if the $(i, j)$ entry of at least one of the matrices $M_{0}, M_{1}, \ldots, M_{m}$ is nonzero, for every $1 \leq i<j \leq n$. The graph $\mathcal{G}$ captures the sparsity of the optimization (20). Observe that those off-diagonal entries of $\widehat{\mathrm{W}}$ that correspond to non-existent edges of $\mathcal{G}$ play no direct role in the SDP relaxation. As a result, it can be inferred that every solution $\mathbf{W}^{\mathrm{opt}}$ to the matrix completion problem (14) or its convex relaxations $(15)$ and $(18)$ is also a solution to the SDP relaxation of the QCQP problem (20). Depending on the choice of $\mathcal{G}^{c}$ in (15) and (18), different low-rank solutions of the SDP relaxation can be generated for a sparse graph $\mathcal{G}$. In particular, there are infinitely many optimization problems with linear objectives, each of which generates a solution $\mathbf{W}^{\text {opt }}$ of the SDP relaxation with rank at most $\operatorname{tw}\{\mathcal{G}\}+1$, provided the optimal tree decomposition of $\mathcal{G}$ is known. Without taking advantage of a tree decomposition, we can generate a solution with rank at most $2(n-\mathrm{OS}(\mathcal{G}))$ in polynomial time (note that this solution can be found efficiently, even though computing the theoretical upper bound on its rank would be an NP-hard problem).

Penalized SDP Relaxation: The strategy delineated above consists of two steps: (i) finding an arbitrary (potentially high-rank) solution $\widehat{\mathbf{W}}$ of the SDP relaxation for QCQP, and (ii) turning the solution into a lower rank solution $\mathbf{W}^{\text {opt }}$ by solving a second convex optimization based on the matrix completion approach. As will become clear, it is advantageous to integrate these two steps. This will be carried out in the sequel. Consider the convex optimization

$$
\begin{array}{cl}
\min _{\mathbf{W} \in \mathbb{S}_{+}^{n}} & \operatorname{trace}\left\{M_{0} \mathbf{W}\right\}+\varepsilon_{1} \operatorname{trace}\{\mathbf{W}\}+\varepsilon_{2} \sum_{(i, j) \in \mathcal{E}^{c}} t_{i j} W_{i j} \\
\text { s.t. } & \operatorname{trace}\left\{M_{k} \mathbf{W}\right\} \leq 0 \quad \text { for } \quad k=1, \ldots, m \\
& W_{11}=1
\end{array}
$$

for a given graph $\mathcal{G}^{c}$, a scalar $\varepsilon_{1}$, and nonzero numbers $\varepsilon_{2}$ and $t_{i j}$ 's. Notice that the objective of this optimization has two penalty terms: (i) a trace term motivated by the nuclear norm technique for rank compensation, and (ii) a weighted sum of some off-diagonal entries of $\mathbf{W}$ motivated by the matrix completion approach described earlier. Assume that Slater's condition holds for Optimization (24) and its dual. As before, every solution $\mathbf{W}^{\mathrm{opt}}$ of the above penalized SDP problem satisfies the inequality

$$
\operatorname{rank}\left\{\mathbf{W}^{\mathrm{opt}}\right\} \leq n-\min _{\mathcal{G}^{s} \subseteq \mathcal{G}} \operatorname{OS}\left(\mathcal{G}^{s} \cup \mathcal{G}^{c}\right)
$$

where the right side of the inequality can be replaced by $t+1$ if $\mathcal{G}^{c}$ is constructed from a tree decomposition of $\mathcal{G}$ with width $t$ such that all nodes are of the identical size. Note that the penalized SDP may become arbitrarily close to the SDP problem by making $\varepsilon_{1}$ sufficiently small or equal to zero. This means that an $\varepsilon$-approximation of a low-rank solution of the SDP relaxation of QCQP can be obtained through the penalized SDP problem. In other words, the proposed penalization eliminates high-rank solutions of the SDP relaxation. A similar penalization technique can be derived based on Optimization (18), leading to the upper bound $2\left(n-\mathrm{OS}\left(\mathcal{G} \cup \mathcal{G}^{c}\right)\right)$ on the rank of all solutions of the corresponding penalized SDP.

Consider a QCQP problem whose underlying sparsity graph $\mathcal{G}$ has a relatively small treewidth. The above penalized convex relaxation generates only low-rank solutions for an infinite choice of coefficients $\varepsilon_{1}, \varepsilon_{2}$ and $t_{i j}$ 's. Our simulations on thousands of energy optimization and decentralized control problems suggest that it is possible to generate a near-global rank-1 solution by meticulously devising $t_{i j}$ 's and tuning the regularization parameters $\varepsilon_{1}$ and $\varepsilon_{2}$ [31], [40], [59], [60].

\section{General Polynomial Optimization}

The preceding subsection provided some results on studying a sparse QCQP based on the graph notions of OS and treewidth. A question arises as to whether the proposed approach can be applied to a dense QCQP or a general polynomial optimization. To address this problem, consider the optimization

$$
\begin{aligned}
\min _{\mathbf{x} \in \mathbb{R}^{n}} & f_{0}(\mathbf{x}) \\
\text { s.t. } & f_{i}(\mathbf{x}) \leq 0, \quad i=1, \ldots, m
\end{aligned}
$$

where $f_{0}, \ldots, f_{m}$ are arbitrary polynomial functions. Assume that the above problem is feasible. This class of optimization includes discrete optimization problems $\left(x_{i}= \pm 1\right.$ can be cast as $x_{i}^{2}=1$ ), and can approximate almost every continuous optimization problem using a Taylor series expansion. The 
above optimization problem can be converted to a QCQP via introducing slack parameters and imposing additional constraints [2]. For instance, the polynomial $x_{1}^{2}+x_{2}^{2} x_{3}$ with three variables can be expressed as $x_{1}^{2}+x_{4} x_{3}$ subject to the additional constraint $x_{2}^{2}=x_{4}$ with a new variable $x_{4}$. This polynomial can also be cast as $x_{1}^{2}+x_{2} x_{4}$ subject to $x_{4}=x_{2} x_{3}$. More precisely, every polynomial optimization can be formulated as a QCQP by increasing the dimension of the problem by a logarithmic factor. This will lead to a QCQP problem of the form (20), but perhaps with no sparsity structure. In what follows, we will explain how the resulting dense QCQP can be sparsified. The details may be found in [61].

Let $\mathcal{G}=(\mathcal{V}, \mathcal{E})$ represent the sparsity graph (i.e., generalized weighted graph) of the QCQP, which may be as sparse as a tree or as dense as a complete graph. Consider a vertex $i$ of the graph $\mathcal{G}$ with degree at least 2 and reformulate the QCQP problem according to the following procedure.

Vertex Duplication Procedure: Perform the following actions:

- Replace the variable $x_{i}$ of QCQP with two new variables $x_{i_{1}}$ and $x_{i_{2}}$.

- Divide the neighboring nodes of vertex $i$ in the graph $\mathcal{G}$ into two arbitrary sets, denoted as $\mathcal{V}_{1}(i)$ and $\mathcal{V}_{2}(i)$

- For every $j$ such that $(i, j) \in \mathcal{E}$, replace all occurrences of the term $x_{i} x_{j}$ in the objective and constraints of the optimization with $x_{i_{1}} x_{j}$ if $j \in \mathcal{V}_{1}(i)$ and with $x_{i_{2}} x_{j}$ if $j \in \mathcal{V}_{2}(i)$.

- Add the additional consistency constraint $x_{i_{1}}=x_{i_{2}}$ to the optimization.

Note that the idea of duplicating a parameter has been extensively utilized in distributed computation. The above procedure modifies the graph $\mathcal{G}$ as follows:

- Vertex $i$ is replaced by two new vertices $i_{1}$ and $i_{2}$.

- Vertices $i_{1}$ and $i_{2}$ are connected to the neighboring subsets $\mathcal{V}_{1}(i)$ and $\mathcal{V}_{2}(i)$, respectively.

- Vertices $i_{1}$ and $i_{2}$ are both connected to vertex 1 to account for the consistency constraint (note that $x_{i_{1}}=$ $x_{i_{2}}$ is indeed equal to $x_{1} x_{i_{1}}=x_{1} x_{i_{2}}$ as $x_{1}$ plays the role of number 1 ).

As can be seen, the above procedure aims to sparsify the graph and reduce its treewidth. In particular, if the above procedure is repeated a sufficient number of times (on the order of the number of edges of $\mathcal{G}$ ), then all edges $(i, j)$ of $\mathcal{G}$ with $i, j \geq 2$ will become isolated and hence the resulting graph will have treewidth $t=2$ (because the removal of vertex 1 in the new graph eliminates all cycles of the resulting graph). This implies that every polynomial optimization can be converted to an equivalent sparse QCQP whose SDP relaxation has a solution $\mathbf{W}^{\text {opt }}$ with rank at most $t+1=3$ (see Subsection III-B. A question arises as to whether there exists a relaxation for which $\operatorname{rank}\left\{\mathbf{W}^{\text {opt }}\right\} \leq 2$. To address this question, consider the following procedure for an edge $(i, j) \in \mathcal{G}$.

Edge Elimination Procedure: Perform the following ac- tions:

- Add two auxiliary variables $z_{1}$ and $z_{2}$.

- Impose the additional constraints:

$$
z_{1}=\frac{x_{i}+x_{j}}{2}, \quad z_{2}=\frac{x_{i}-x_{j}}{2}
$$

- Replace every instance of the product $x_{i} x_{j}$ in the QCQP problem with $z_{1}^{2}-z_{2}^{2}$.

The above procedure eliminates the edge $(i, j)$ and its repetition makes the resulting graph have treewidth $t=1$. Hence, the SDP relaxation corresponding to the obtained sparse QCQP has a matrix solution with rank 1 or 2. This result has two implications:

i) The NP-hardness of various subclasses of polynomial optimization, e.g., combinatorial optimization, is only related to the existence of a not rank-1 but low-rank SDP solution, where the upper bound on the rank is constant and does not depend on the size of the original optimization.

ii) By approximating the low-rank solution of the SDP relaxation with a rank-1 matrix, an approximate solution of the original problem may be obtained whose closeness to the global solution can also be upper bounded.

These results offer a new insight into the computational complexity of polynomial optimization (Property (i)) and enable to seek a near-global solution (Property (ii)).

There are two important parameters associated with an SDP relaxation: (i) optimal cost serving as a lower bound on the optimal value of the QCQP, and (ii) minimum rank of the SDP solution. It can be shown that the proposed sparsification technique leads to a non-unique hierarchy of SDP relaxations, which does not improve the lower bound but reduces the rank. In general, since there are many ways to sparsify the graph (by determining which vertex of $\mathcal{G}$ to choose and how to partition its edges), it is imperative to perform the sparsification in such a way that the optimal value of SDP relaxation is not decreased noticeably and yet the rank is improved significantly.

Assume that we have designed an SDP relaxation possessing a low-rank matrix solution $\mathbf{W}^{\text {opt }}$. Now, three strategies could be taken to find a near-global (sub-optimal) solution of the original QCQP:

- Since $\mathbf{W}^{\text {opt }}$ has only a few undesirable (nonzero) eigenvalues, it may be converted to an approximate solution via a local search algorithm. Based on the eigenvalue decomposition, it is straightforward to design an iterative algorithm with the property that the rank of the solution does not increase at any iteration of the algorithm. This leads to a sequence of low-rank matrices, which tends to converge to a rank-1 solution. The obtained solution may ultimately need to be approximated by a rank-1 matrix if it is not ultimately rank-1.

- The unwanted nonzero eigenvalues of $\mathbf{W}^{\text {opt }}$ may be eliminated by means of a penalization technique such as the one mentioned earlier. 
- Another technique is to directly approximate $\mathbf{W}^{\mathrm{opt}}$ with a rank-1 matrix by solving a convex optimization.

\section{CASE Study: Optimal Distributed Control}

Consider the problem of designing an optimal distributed controller for a multi-channel deterministic or stochastic system, where the optimality is measured with respect to a linear-quadratic, $H_{2}$, or $H_{\infty}$ performance index. It has been long known that this problem is computationally hard and, in particular NP-hard in the worst case [62]-[64]. Great effort has been devoted to investigating this highly complex problem for special types of systems, including spatially distributed systems [65]-[69], dynamically decoupled systems [70], [71], weakly coupled systems [72], and strongly connected systems [73]. Another special case that has received considerable attention is the design of an optimal static distributed controller [74], [75]. Early approaches for the optimal decentralized control problem were based on parameterization techniques [76], [77], which were then evolved into matrix optimization methods [78], [79].

Due to the recent advances in the area of convex optimization, the focus of the existing research efforts has shifted from deriving a closed-form solution for the above control synthesis problem to finding a convex formulation of the problem that can be efficiently solved numerically [80][82]. This has been carried out in the seminal work [83] by deriving a sufficient condition named quadratic invariance, which has been generalized in [84] by deploying the concept of partially order sets. These conditions have been further investigated in several other papers [85]-[87]. A different approach is taken in the recent papers [88], [89], where it has been shown that the distributed control problem can be cast as a convex optimization for positive systems.

\section{A. Time-Domain Formulation}

Consider the linear discrete-time system

$$
\left\{\begin{array}{rl}
x[\tau+1] & =A x[\tau]+B u[\tau] \\
y[\tau] & =C x[\tau]
\end{array}, \quad \forall \tau \in \mathbb{Z}_{+}\right.
$$

where $x[\tau] \in \mathbb{R}^{n}, u[\tau] \in \mathbb{R}^{m}$ and $y[\tau] \in \mathbb{R}^{r}$ denote the state, input and output of the system, respectively. The system matrices $A, B, C$ and the initial state $x[0]$ are all known. The goal is to design a fixed-order dynamic controller with a pre-specified structure to minimize the cost function $\sum_{\tau=0}^{p}\left(x[\tau]^{*} Q x[\tau]+u[\tau]^{*} R u[\tau]\right)$ for an arbitrary terminal time $p$ and positive definite matrices $Q$ and $R$. To solve this optimal distributed control (ODC) problem, we denote the unknown controller as

$$
\left\{\begin{array}{rl}
z[\tau+1] & =A_{c} z[\tau]+B_{c} y[\tau] \\
u[\tau] & =C_{c} z[\tau]+D_{c} y[\tau]
\end{array}, \quad \forall \tau \in \mathbb{Z}_{+}\right.
$$

where $z[\tau] \in \mathbb{R}^{n_{c}}$ represents the state of the controller, $n_{c}$ denotes its known degree, and the quadruple $\left(A_{c}, B_{c}, C_{c}, D_{c}\right)$ needs to be designed. Since the controller is required to have a pre-determined distributed structure, the 4-tuple $\left(A_{c}, B_{c}, C_{c}, D_{c}\right)$ must belong to some given polytope $\mathcal{K}$. This polytope enforces certain entries of $A_{c}, B_{c}, C_{c}$, and

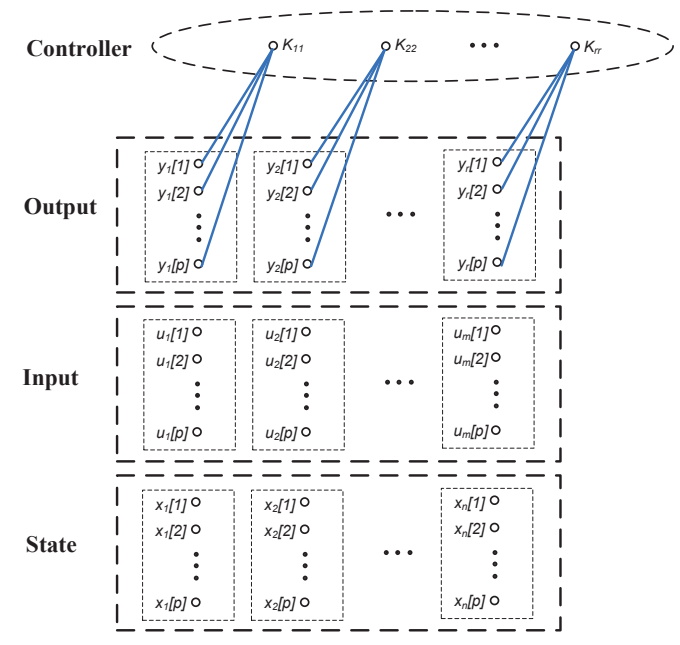

Fig. 6: The graph showing the sparse nonlinearity of ODC for the time-domain formulation (node 1 is not shown in the graph due to its connection to almost all nodes of the graph).

$D_{c}$ to be zero. ODC is a nonlinear optimization because the dynamics of the controller has some non-convex terms such as $A_{c} z[\tau]$ and $B_{c} y[\tau]$. In order to solve the above ODC problem, define:

$$
\begin{array}{r}
\mathbf{v}:=\left[\begin{array}{cccccccc}
1 & h^{*} & x[0]^{*} & \cdots & x[p]^{*} & y[0]^{*} & \cdots & y[p]^{*} \\
& u[0]^{*} & \cdots & u[p]^{*} & z[0]^{*} & \cdots & z[p]^{*}
\end{array}\right]^{*}
\end{array}
$$

where $h$ denotes a vector consisting of all free (nonzero) entries of the matrices $A_{c}, B_{c}, C_{c}$, and $D_{c}$. The ODC problem can be cast as a quadratic optimization with respect to the vector $\mathbf{v}$ and as a linear optimization in the matrix $\mathbf{v v}^{*}$. Hence, an SDP relaxation of this problem can be derived by replacing $\mathbf{v v}^{*}$ with a new matrix variable $\mathbf{W}$.

\section{B. Lyapunov-Domain Formulation}

The previous formulation of the ODC problem was restricted to deterministic systems. Consider now the stochastic system

$$
\left\{\begin{array}{rl}
x[\tau+1] & =A x[\tau]+B u[\tau]+E d[\tau] \\
y[\tau] & =C x[\tau]+F v[\tau]
\end{array}, \quad \tau \in \mathbb{Z}_{+}\right.
$$

where $d[\tau]$ and $v[t]$ are random variables representing the system's disturbance and measurement noise, which are assumed to be zero-mean white-noise random processes. The objective is to design a distributed controller with a prespecified structure for the above system to minimize the cost function

$$
\lim _{\tau \rightarrow+\infty} \mathcal{E}\left(x[\tau]^{*} Q x[\tau]+u[\tau]^{*} R u[\tau]\right)
$$

where $\mathcal{E}\{\cdot\}$ shows the expectation operator. Since we can no longer formulate ODC as a QCQP with respect to the timedomain signals (due to unknown disturbances), we resort to a bilinear matrix formulation in terms of Lyapunov matrices. 
To explain the idea, consider the special case of designing a static controller. Define two covariance matrices as below:

$$
\Sigma_{d}=\mathcal{E}\left\{E d[0] d[0]^{*} E^{*}\right\}, \quad \Sigma_{v}=\mathcal{E}\left\{F v[0] v[0]^{*} F^{*}\right\}
$$

The problem of designing an optimal static structured controller $u[t]=K y[t]$ for the system $[30$ to minimize $[31$ can be formulated as the minimization of

$$
\operatorname{trace}\left\{P \Sigma_{d}+M \Sigma_{v}+K^{*} R K \Sigma_{v}\right\}
$$

subject to the constraints

$$
\begin{aligned}
& {\left[\begin{array}{cccc}
G & G & (A G+B L)^{*} & L^{*} \\
G & Q^{-1} & 0 & 0 \\
A G+B L & 0 & G & 0 \\
L & 0 & 0 & R^{-1}
\end{array}\right] \succeq 0,} \\
& {\left[\begin{array}{cc}
P & I \\
I & G
\end{array}\right] \succeq 0,} \\
& {\left[\begin{array}{cc}
M & (B K)^{*} \\
B K & G
\end{array}\right] \succeq 0,} \\
& L=K C G
\end{aligned}
$$

where $K$ is a matrix variable with forced zeros in certain entries, $P$ is an unknown Lyapunov matrix, and $G \in \mathbb{S}^{n}$, $L \in \mathbb{R}^{n \times r}$ and $M \in \mathbb{S}^{r}$ are auxiliary matrices. The above problem is non-convex due to the nonlinear term $L=K C G$. To convexify the problem, define $\mathbf{v}$ as a vector consisting of number $1, h$, and all entries of the matrix $G$. Then, every entry of $K C G$ can be expressed as a quadratic function of $\mathbf{v}$. Hence, the above optimization has a natural SDP relaxation with a variable $\mathbf{W}$ playing the role of $\mathbf{v v}^{*}$.

\section{Existence of Low-Rank Solution}

Two quadratic formulations of the ODC problem have been proposed for deterministic and stochastic systems. The graphs capturing the sparsity of these problems are both extremely sparse with small treewidth numbers. For example, in the special case of designing a fully decentralized static controller (diagonal $K$ ), the graph for the time-domain formulation reduces to a bunch of stars after removing its node 1. This fact is illustrated in Figure 6. As a result, the treewidth of this graph is equal to 2, which implies that the SDP relaxation of the ODC problem has a lowrank solution with rank at most 3 under this circumstance. The same conclusion holds for the Lyapunov-domain formulation. Extensive simulations have been conducted in [40], [59], [60], where it has been verified on random and physical systems that the rank-3 solution can be well approximated by a rank-1 matrix. For instance, several hundred random systems were generated in those papers for which nearoptimal decentralized controllers with a global optimality degree of at least $99 \%$ were designed. The same observation was made for the distributed control of power grids. The reader may refer to [59], [60] to see how the numerical complexity of the proposed SDP relaxations may also be reduced significantly, leading to solving computationallycheap SDP relaxations.

\section{Case Study: Power Optimization Problems}

In the past five decades, many optimization techniques have been studied for the non-convex optimal power flow (OPF) problem, including: linear programming, Newton Raphson, quadratic programming, nonlinear programming, Lagrange relaxation, interior point methods, artificial intelligence, artificial neural network, fuzzy logic, genetic algorithm, evolutionary programming, and particle swarm optimization [90]-[102]. Most of these methods are built on the Karush-Kuhn-Tucker (KKT) necessary conditions, which can only find a local solution (as opposed to a globally optimal solution). Some recent efforts have been focused on convex optimization [103]-[105]. The practical difficulty of treating the non-linearity of OPF has resulted in most optimization formulations resorting to approximations such as linearization. The problem of discovering new approaches for avoiding linearization has received a special attention by Federal Energy Regulatory Commission in the past few years [106]-[109].

\section{A. Problem Formulation}

Consider an $n$-bus power network described by a graph $\mathcal{G}=(\mathcal{V}, \mathcal{E})$, where each vertex belonging to $\mathcal{V}=\{1, \ldots, n\}$ represents a node (bus) of the network and each edge belonging to $\mathcal{E}$ represents a transmission line. Let $y_{i j}$ denote the admittance of the line $(i, j) \in \mathcal{E}$. Define $\mathbf{V} \in \mathbb{C}^{n}$ as the voltage phasor vector where its component $V_{k}$ represents the complex voltage at node $k \in \mathcal{V}$. Assume that each node of the network is connected to a known load as well as a generator with an unknown production level. OPF is a resource allocation problem, which aims to optimize the production levels of the generators. To formulate the problem, let $P_{k}$ and $Q_{k}$ denote the net active and reactive powers injected at node $k \in \mathcal{G}$ (net power is equal to generation minus load). OPF can be expressed as:

$$
\begin{aligned}
& \min _{\mathbf{V}, \mathbf{P}, \mathbf{Q} \in \mathbb{C}^{n}} \sum_{k \in \mathcal{V}} f_{k}\left(P_{k}\right) \\
& \text { s.t. } \quad V_{k}^{\min } \leq\left|V_{k}\right| \leq V_{k}^{\max }, \quad k \in \mathcal{V} \\
& P_{k}^{\min } \leq P_{k} \leq P_{k}^{\max }, \quad k \in \mathcal{V} \\
& Q_{k}^{\min } \leq Q_{k} \leq Q_{k}^{\max }, \quad k \in \mathcal{V} \\
& \operatorname{Re}\left\{V_{i}\left(V_{i}^{*}-V_{j}^{*}\right) y_{i j}^{*}\right\} \leq P_{i j}^{\max },(i, j) \in \mathcal{E} \\
& P_{k}+Q_{k} \sqrt{-1}=\sum_{i \in \mathcal{N}(k)} V_{k}\left(V_{k}^{*}-V_{i}^{*}\right) y_{k i}^{*}, k \in \mathcal{V}
\end{aligned}
$$

where $V_{k}^{\min }, V_{k}^{\max }, P_{k}^{\min }, P_{k}^{\max }, Q_{k}^{\min }, Q_{k}^{\max }$ and $P_{i j}^{\max }$ are network limits, $\mathcal{N}(k)$ denotes the set of neighbors of vertex $k$, and $f_{k}\left(S_{k}\right)$ is a convex function accounting for the power generation cost at node $k$.

\section{B. Existence of Low-Rank Solution}

Problem (35) is quadratic in the vector V. Therefore, an SDP relaxation of OPF can be derived by reformulating the problem in terms of $\mathbf{V V}^{*}$ and then replacing $\mathbf{V} \mathbf{V}^{*}$ with a new variable W. It is known that (see [27]-[30], [32], [110][115]): 

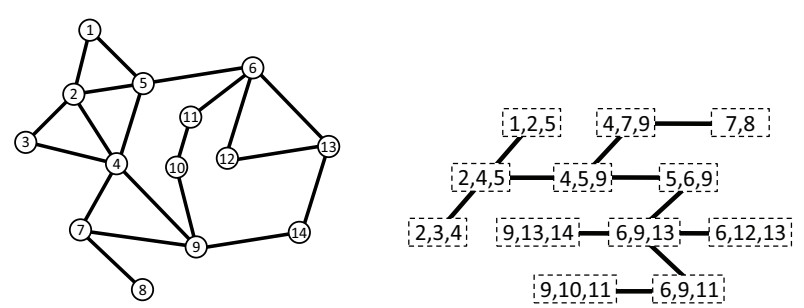

Fig. 7: The IEEE 14-bus test case and its minimal tree decomposition

- The SDP relaxation is exact for IEEE benchmark systems with 14, 30, 57, 118 and 300 buses, several Polish systems, and many randomly generated power networks. This technique is the first method proposed since the introduction of the OPF problem in 1962 that is able to find a provably global solution for certain OPF problems.

- Under some practical assumptions, the SDP relaxation is exact for distribution networks (acyclic graphs). However, it may not be exact for transmission networks (cyclic graphs) unless there is a sufficient number of transformers in the network.

- There are OPF problems defined over transmission networks for which the SDP exact is not exact but a penalized SDP relaxation works.

- The sign definite condition stated before holds for power networks and this is one of the main reasons behind the success of the SDP relaxation for OPF.

Since the SDP relaxation is not always exact for OPF over transmission networks, it is highly desirable to seek a nearglobal solution. We have calculated the treewidth of $\mathcal{G}$ for several power systems and reported our findings in [115]. It can be seen that the treewidth of a Polish network with 3375 nodes is at most 28 . Figure 7 shows a minimal tree decomposition associated with the IEEE 14 case. As long as the treewidth is small, the SDP relaxation of OPF will have a low-rank solution for transmission networks, which may be leveraged to design a near-global solution. This idea has been tested on 7000 instances of OPF in [32], [115].

\section{CONCLuSions}

This tutorial paper aims to study an arbitrary non-convex polynomial optimization problem through semidefinite programming (SDP) relaxations combined with graph-theoretic algorithms. Three problems are investigated in detail. First, a method is proposed to study how the underlying structure of an optimization problem reduces the computational complexity of the problem. For this purpose, the structure of the optimization is mapped into a weighted graph and it is shown that the SDP relaxation is exact if the graph possesses certain properties. Second, it is shown that the SDP relaxation of a sparse optimization problem has a solution whose rank can be characterized in terms of the sparsity level of the problem. Third, it is explained that every polynomial optimization problem admits a sparse representation whose
SDP relaxation has a rank 1 or 2 matrix solution. Two engineering applications of these results are also discussed.

\section{REFERENCES}

[1] S. Boyd and L. Vandenberghe, Convex Optimization. Cambridge, 2004.

[2] Y. Nesterov, A. S. Nemirovskii, and Y. Ye, Interior-point polynomial algorithms in convex programming. SIAM, 1994.

[3] D. P. Bertsekas, "Nonlinear programming," 1999.

[4] A. Ben-Tal and A. S. Nemirovski, Lectures on modern convex optimization: analysis, algorithms, and engineering applications. SIAM, 2001.

[5] K. G. Murty and S. N. Kabadi, "Some NP-complete problems in quadratic and nonlinear programming," Mathematical programming, vol. 39, no. 2, pp. 117-129, 1987.

[6] E. D. Klerk, "The complexity of optimizing over a simplex, hypercube or sphere: a short survey," Central European Journal of Operations Research, vol. 16, pp. 111-125, 2008.

[7] G. L. Nemhauser and L. A. Wolsey, Integer and combinatorial optimization. Wiley New York, 1988.

[8] C. H. Papadimitriou and K. Steiglitz, Combinatorial optimization: algorithms and complexity. Courier Dover Publications, 1998.

[9] S. Boyd, L. E. Ghaoui, E. Feron, and V. Balakrishnan, "Linear matrix inequalities in system and control theory," Studies in Applied Mathematics, SIAM, 1994.

[10] M. X. Goemans and D. P. Williamson, "Improved approximation algorithms for maximum cut and satisfiability problems using semidefinite programming," Journal of the ACM (JACM), vol. 42, no. 6, pp. 1115-1145, 1995.

[11] J. Briet, F. Vallentin et al., "Grothendieck inequalities for semidefinite programs with rank constraint," arXiv preprint arXiv:1011.1754, 2010.

[12] N. Alon, K. Makarychev, Y. Makarychev, and A. Naor, "Quadratic forms on graphs," Inventiones mathematicae, vol. 163, no. 3, pp. 499-522, 2006.

[13] M. Laurent and A. Varvitsiotis, "Computing the grothendieck constant of some graph classes," Operations Research Letters, vol. 39, no. 6, pp. 452-456, 2011.

[14] M. X. Goemans and D. P. Williamson, "Approximation algorithms for max-3-cut and other problems via complex semidefinite programming," Journal of Computer and System Sciences, vol. 68, pp. 422470, 2004.

[15] Y. Nesterov, "Semidefinite relaxation and nonconvex quadratic optimization," Optimization Methods and Software, vol. 9, pp. 141-160, 1998.

[16] Y. Ye, "Approximating quadratic programming with bound and quadratic constraints," Mathematical Programming, vol. 84, pp. 219226, 1999.

[17] Y. Ye, "Approximating global quadratic optimization with convex quadratic constraints," Journal of Global Optimization, vol. 15, pp. $1-17,1999$.

[18] S. Zhang, "Quadratic maximization and semidefinite relaxation," Mathematical Programming A, vol. 87, pp. 453-465, 2000.

[19] S. Zhang and Y. Huang, "Complex quadratic optimization and semidefinite programming," SIAM Journal on Optimization, vol. 87, pp. 871-890, 2006.

[20] Z. Luo, N. Sidiropoulos, P. Tseng, and S. Zhang, "Approximation bounds for quadratic optimization with homogeneous quadratic constraints," SIAM Journal on Optimization, vol. 18, pp. 1-28, 2007.

[21] S. He, Z. Luo, J. Nie, and S. Zhang, "Semidefinite relaxation bounds for indefinite homogeneous quadratic optimization," SIAM Journal on Optimization, vol. 19, pp. 503-523, 2008.

[22] S. He, Z. Li, and S. Zhang, "Approximation algorithms for homogeneous polynomial optimization with quadratic constraints," Mathematical Programming, vol. 125, pp. 353-383, 2010.

[23] J. B. Lasserre, "An explicit exact SDP relaxation for nonlinear 0-1 programs," in Integer Programming and Combinatorial Optimization. Springer, 2001, pp. 293-303.

[24] S. Kim and M. Kojima, "Exact solutions of some nonconvex quadratic optimization problems via SDP and SOCP relaxations," Computational Optimization and Applications, vol. 26, no. 2, pp. 143-154, 2003. 
[25] S. Sojoudi and J. Lavaei, "On the exactness of semidefinite relaxation for nonlinear optimization over graphs: Part I," IEEE Conference on Decision and Control, 2013.

[26] S. Sojoudi and J. Lavaei, "On the exactness of semidefinite relaxation for nonlinear optimization over graphs: Part II," IEEE Conference on Decision and Control, 2013.

[27] J. Lavaei and S. H. Low, "Zero duality gap in optimal power flow problem," IEEE Transactions on Power Systems, vol. 27, no. 1, pp. 92-107, 2012.

[28] J. Lavaei, "Zero duality gap for classical OPF problem convexifies fundamental nonlinear power problems," American Control Conference, 2011.

[29] S. Sojoudi and J. Lavaei, "Physics of power networks makes hard optimization problems easy to solve," IEEE Power \& Energy Society General Meeting, 2012.

[30] J. Lavaei, B. Zhang, and D. Tse, "Geometry of power flows in tree networks," IEEE Power \& Energy Society General Meeting, 2012.

[31] S. Sojoudi, R. Madani, and J. Lavaei, "Low-rank solution of convex relaxation for optimal power flow problem," IEEE SmartGridComm, 2013.

[32] R. Madani, S. Sojoudi, and J. Lavaei, "Convex relaxation for optimal power flow problem: Mesh networks," To appear in IEEE Transactions on Power Systems, 2014.

[33] M. Fazel, H. Hindi, and S. Boyd, "Log-det heuristic for matrix rank minimization with applications to hankel and euclidean distance matrices," American Control Conference, vol. 3, pp. 2156-2162, 2003.

[34] B. Recht, M. Fazel, and P. A. Parrilo, "Guaranteed minimum rank solutions to linear matrix equations via nuclear norm minimization,' SIAM Review, vol. 52, pp. 471-501, 2010.

[35] A. Barvinok, "Problems of distance geometry and convex properties of quadartic maps," Discrete and Computational Geometry, vol. 12, pp. 189-202, 1995.

[36] G. Pataki, "On the rank of extreme matrices in semidefinite programs and the multiplicity of optimal eigenvalues," Mathematics of Operations Research, vol. 23, pp. 339-358, 1998.

[37] A. Barvinok, "A remark on the rank of positive semidefinite matrices subject to affine constraints," Discrete \& Computational Geometry, vol. 25, no. 1, pp. 23-31, 2001.

[38] W. Ai, Y. Huang, and S. Zhang, "On the low rank solutions for linear matrix inequalities," Mathematics of Operations Research, vol. 33, no. 4, pp. 965-975, 2008.

[39] S. Sojoudi and J. Lavaei, "Exactness of semidefinite relaxations for nonlinear optimization problems with underlying graph structure," to appear in SIAM Journal on Optimization, 2014.

[40] J. Lavaei, "Optimal decentralized control problem as a rankconstrained optimization," Allerton, 2013.

[41] J. F. Sturm and S. Zhang, "On cones of nonnegative quadratic functions," Mathematics of Operations Research, vol. 28, pp. 246267, 2003.

[42] Y. Huang and S. Zhang, "Complex matrix decomposition and quadratic programming," Mathematics of Operations Research, vol. 32, pp. 758-768, 2007.

[43] W. Ai, Y. Huang, and S. Zhang, "On the low rank solutions for linear matrix inequalities," Mathematics of Operations Research, vol. 33, pp. 965-975, 2008.

[44] S. Kim and M. Kojima, "Exact solutions of some nonconvex quadratic optimization problems via SDP and SOCP relaxations," Computational Optimization and Applications, vol. 26, p. 143154, 2003.

[45] S. Bose and K. M. C. D. F. Gayme, S. H. Low, "Quadratically constrained quadratic programs on acyclic graphs with application to power flow," arXiv:1203.5599v1, 2012.

[46] M. Padberg, "The boolean quadric polytope: some characteristics, facets and relatives," Mathematical programming, vol. 45, no. 1-3, pp. 139-172, 1989.

[47] P. A. Parrilo and B. Sturmfels, "Minimizing polynomial functions," Algorithmic and quantitative real algebraic geometry, DIMACS Series in Discrete Mathematics and Theoretical Computer Science, vol. 60, pp. 83-99, 2003.

[48] R. Madani, G. Fazelnia, S. Sojoudi, and J. Lavaei, "Low-rank solutions of matrix inequalities with applications to polynomial optimization and matrix completion problems," Conference on Decision and Control, 2014.
[49] P. Hackney, B. Harris, M. Lay, L. H. Mitchell, S. K. Narayan, and A. Pascoe, "Linearly independent vertices and minimum semidefinite rank," Linear Algebra and its Applications, vol. 431, no. 8, pp. 11051115, 2009.

[50] M. Laurent and A. Varvitsiotis, "A new graph parameter related to bounded rank positive semidefinite matrix completions," Mathematical Programming, pp. 1-35, 2012.

[51] S. Arnborg, D. G. Corneil, and A. Proskurowski, "Complexity of finding embeddings in ak-tree," SIAM Journal on Algebraic Discrete Methods, vol. 8, no. 2, pp. 277-284, 1987.

[52] J. Matoušek and R. Thomas, "Algorithms finding tree-decompositions of graphs," Journal of Algorithms, vol. 12, no. 1, pp. 1-22, 1991.

[53] H. L. Bodlaender, "A linear-time algorithm for finding treedecompositions of small treewidth," SIAM Journal on computing, vol. 25, no. 6, pp. 1305-1317, 1996.

[54] S. M. Fallat and L. Hogben, "The minimum rank of symmetric matrices described by a graph: a survey," Linear Algebra and its Applications, vol. 426, no. 2, pp. 558-582, 2007.

[55] W. Barrett, H. van der Holst, and R. Loewy, "Graphs whose minimal rank is two," Electronic Journal of Linear Algebra, vol. 11, no. 258280, p. 687, 2004.

[56] J. Sinkovic and H. van der Holst, "The minimum semidefinite rank of the complement of partial k-trees," Linear Algebra and its Applications, vol. 434, no. 6, pp. 1468-1474, 2011.

[57] L. Vandenberghe and S. Boyd, "Semidefinite programming," SIAM review, vol. 38, no. 1, pp. 49-95, 1996.

[58] K. M. Anstreicher, "On convex relaxations for quadratically constrained quadratic programming," Mathematical programming, vol. 136, no. 2, pp. 233-251, 2012.

[59] G. Fazelnia, R. Madani, and J. Lavaei, "Convex relaxation for optimal distributed control problem," Conference on Decision and Control, 2014.

[60] A. Kalbat, R. Madani, G. Fazelnia, and J. Lavaei, "Efficient convex relaxation for stochastic optimal distributed control problem," Allerton, 2014.

[61] R. Madani, G. Fazelnia, and J. Lavaei, "Rank-2 matrix solution for semidefinite relaxations of arbitrary polynomial optimization problems," 2014. [Online]. Available: http://www.ee.columbia.edu/ $\sim$ lavaei/CDC_Polynomial_2014.pdf

[62] H. Witsenhausen, "A counterexample in stochastic optimum control," SIAM Journal on Control, vol. 6, no. 1, pp. 131-147, 1968.

[63] C. Papadimitriou and J. Tsitsiklis, "Intractable problems in control theory," SIAM Journal on Control and Optimization, vol. 24, no. 4, pp. 639-654, 1986.

[64] V. D. Blondel and J. N. Tsitsiklis, "A survey of computational complexity results in systems and control," Automatica, vol. 36, no. 9, pp. 1249 - 1274, 2000.

[65] R. D'Andrea and G. Dullerud, "Distributed control design for spatially interconnected systems," IEEE Transactions on Automatic Control, vol. 48, no. 9, pp. 1478-1495, 2003.

[66] B. Bamieh, F. Paganini, and M. A. Dahleh, "Distributed control of spatially invariant systems," IEEE Transactions on Automatic Control, vol. 47, no. 7, pp. 1091-1107, 2002.

[67] C. Langbort, R. Chandra, and R. D'Andrea, "Distributed control design for systems interconnected over an arbitrary graph," IEEE Transactions on Automatic Control, vol. 49, no. 9, pp. 1502-1519, 2004.

[68] N. Motee and A. Jadbabaie, "Optimal control of spatially distributed systems," IEEE Transactions on Automatic Control, vol. 53, no. 7, pp. 1616-1629, 2008.

[69] G. Dullerud and R. D’Andrea, "Distributed control of heterogeneous systems," IEEE Transactions on Automatic Control, vol. 49, no. 12, pp. 2113-2128, 2004.

[70] T. Keviczky, F. Borrelli, and G. J. Balas, "Decentralized receding horizon control for large scale dynamically decoupled systems," Automatica, vol. 42, no. 12, pp. 2105-2115, 2006.

[71] F. Borrelli and T. Keviczky, "Distributed LQR design for identical dynamically decoupled systems," IEEE Transactions on Automatic Control, vol. 53, no. 8, pp. 1901-1912, 2008.

[72] D. D. Siljak, "Decentralized control and computations: status and prospects," Annual Reviews in Control, vol. 20, pp. 131-141, 1996.

[73] J. Lavaei, "Decentralized implementation of centralized controllers for interconnected systems," IEEE Transactions on Automatic Control, vol. 57, no. 7, pp. 1860-1865, 2012. 
[74] M. Fardad, F. Lin, and M. R. Jovanovic, "On the optimal design of structured feedback gains for interconnected systems," in 48th IEEE Conference on Decision and Control, 2009, pp. 978-983.

[75] F. Lin, M. Fardad, and M. R. Jovanovic, "Augmented lagrangian approach to design of structured optimal state feedback gains," IEEE Transactions on Automatic Control, vol. 56, no. 12, pp. 2923-2929, 2011.

[76] J. Geromel, J. Bernussou, and P. Peres, "Decentralized control through parameter space optimization," Automatica, vol. 30, no. 10, pp. 1565 - 1578, 1994.

[77] R. A. Date and J. H. Chow, "A parametrization approach to optimal $\mathrm{H}_{2}$ and $\mathrm{H}_{\infty}$ decentralized control problems," Automatica, vol. 29, no. 2, pp. 457 - 463, 1993.

[78] G. Scorletti and G. Duc, "An LMI approach to dencentralized $H_{\infty}$ control," International Journal of Control, vol. 74, no. 3, pp. 211224, 2001.

[79] G. Zhai, M. Ikeda, and Y. Fujisaki, "Decentralized $H_{\infty}$ controller design: a matrix inequality approach using a homotopy method,' Automatica, vol. 37, no. 4, pp. 565 - 572, 2001.

[80] G. A. de Castro and F. Paganini, "Convex synthesis of localized controllers for spatially invariant systems," Automatica, vol. 38, no. 3, pp. $445-456,2002$.

[81] B. Bamieh and P. G. Voulgaris, "A convex characterization of distributed control problems in spatially invariant systems with communication constraints," Systems \& Control Letters, vol. 54, no. 6, pp. $575-583,2005$

[82] X. Qi, M. Salapaka, P. Voulgaris, and M. Khammash, "Structured optimal and robust control with multiple criteria: a convex solution," IEEE Transactions on Automatic Control, vol. 49, no. 10, pp. 16231640, 2004.

[83] M. Rotkowitz and S. Lall, "A characterization of convex problems in decentralized control," IEEE Transactions on Automatic Control, vol. 51, no. 2, pp. 274-286, 2006

[84] P. Shah and P. A. Parrilo, " $\mathrm{H}_{2}$-optimal decentralized control over posets: a state-space solution for state-feedback," http://arxiv.org/ abs/1111.1498 2011.

[85] L. Lessard and S. Lall, "Optimal controller synthesis for the decentralized two-player problem with output feedback," American Control Conference, 2012.

[86] A. Lamperski and J. C. Doyle, "Output feedback $H_{2}$ model matching for decentralized systems with delays," American Control Conference, 2013.

[87] M. Rotkowitz and N. Martins, "On the nearest quadratically invariant information constraint," IEEE Transactions on Automatic Control, vol. 57, no. 5, pp. 1314-1319, 2012.

[88] T. Tanaka and C. Langbort, "The bounded real lemma for internally positive systems and $\mathrm{H}$-infinity structured static state feedback," IEEE Transactions on Automatic Control, vol. 56, no. 9, pp. 2218-2223, 2011.

[89] A. Rantzer, "Distributed control of positive systems," http://arxiv. org/abs/1203.0047 2012.

[90] J. Carpentier, "Contribution to the economic dispatch problem," Bulletin Society Francaise Electriciens, 1962.

[91] R. Baldick, Applied Optimization: Formulation and Algorithms for Engineering Systems. Cambridge, 2006.

[92] M. Huneault and F. Galiana, "A survey of the optimal power flow literature," IEEE Transactions on Power Systems, vol. 6, no. 2, pp. 762-770, 1991

[93] R. A. Jabr, A. H. Coonick, and B. J. Cory, "A primal-dual interior point method for optimal power flow dispatching," IEEE Transactions on Power Systems, vol. 17, no. 3, pp. 654-662, 2002.

[94] G. L. Torres and V. H. Quintana, "Optimal power flow by a nonlinear complementarity method," IEEE Transactions on Power Systems, vol. 15, no. 3, pp. 1028-1033, 2000.

[95] H. Wang, C. E. Murillo-Sanchez, R. D. Zimmerman, and R. J. Thomas, "On computational issues of market-based optimal power flow," IEEE Transactions on Power Systems, vol. 22, no. 3, pp. 1185 1193, 2007.

[96] J. A. Momoh, M. E. El-Hawary, and R. Adapa, "A review of selected optimal power flow literature to 1993. Part I: Nonlinear and quadratic programming approaches," IEEE Transactions on Power Systems, 1999.

[97] J. A. Momoh, M. E. El-Hawary, and R. Adapa, "A review of selected optimal power flow literature to 1993. Part II: Newton, linear programming and interior point methods," IEEE Transactions on Power Systems, 1999.

[98] H. Wei, H. Sasaki, J. Kubokawa, and R. Yokoyama, "An interior point nonlinear programming for optimal power flow problems with a novel data structure," IEEE Transactions on Power Systems, vol. 13 no. 3, pp. 870-877, 1998.

[99] W. M. Lin, C. H. Huang, and T. S. Zhan, "A hybrid current-power optimal power flow technique," IEEE Transactions on Power Systems, vol. 23, no. 1, pp. 177-185, 2008.

[100] Q. Y. Jiang, H. D. Chiang, C. X. Guo, and Y. J. Cao, "Power-current hybrid rectangular formulation for interior-point optimal power flow,' IET Generation, Transmission \& Distribution, vol. 3, no. 8, pp. 748756, 2009.

[101] K. S. Pandya and S. K. Joshi, "A survey of optimal power flow methods," Journal of Theoretical and Applied Information Technology, 2008.

[102] H. D. Chiang and M. E. Baran, "On the existence and uniqueness of load flow solution for radial distribution power networks," IEEE Transactions on Circuits and Systems, vol. 37, no. 3, pp. 410-416, 1990.

[103] R. A. Jabr, "Radial distribution load flow using conic programming," IEEE Transactions on Power Systems, vol. 21, no. 3, pp. 1458-1459, 2006.

[104] K. F. X. Bai, H. Wei and Y. Wang, "Semidefinite programming for optimal power flow problems," International Journal of Electric Power \& Energy Systems, vol. 30, no. 6-7, pp. 383-392, 2008.

[105] R. A. Jabr, "Optimal power flow using an extended conic quadratic formulation," IEEE Transactions on Power Systems, vol. 23, no. 3 , pp. 1000-1008, 2008.

[106] A. Schecter and R. P. O'Neill, "Exploration of the ACOPF feasible region for the standard IEEE test set optimal power flow," 2013, available on FERC website at http://www.ferc.gov/industries/electric/indus-act/market-planning/ opf-papers/acopf-6-test-problem-properties.pdf

[107] P. A. Lipka, R. P. O'Neill, and S. Oren, "Developing line current magnitude constraints for IEEE test problems," 2013, available on FERC website at http://www.ferc.gov/industries/electric/indus-act/ market-planning/opf-papers/acopf-7-line-constraints.pdf

[108] M. Pirnia, R. P. O’Neill, P. A. Lipka, and C. Campaigne, "A computational study of linear approximations to the convex constraints in the iterative linear IV-ACOPF formulation," 2013, available on FERC website at http: //www.ferc.gov/industries/electric/indus-act/market-planning/ opf-papers/acopf-8-preprocessed-constraints-iliv-acopf.pdf

[109] C. Campaigne, P. A. Lipka, M. Pirnia, R. P. O’Neill, and S. Oren, "Testing step-size limits for solving the linearized current voltage AC optimal power flow," 2013, available on FERC website at http://www.ferc.gov/industries/electric/indus-act/market-planning/ opf-papers/acopf-9-stepsizelimits-iliv-acopf.pdf

[110] J. Lavaei and S. H. Low, "Convexification of optimal power flow problem," 48th Annual Allerton Conference, 2010.

[111] J. Lavaei, A. Rantzer, and S. H. Low, "Power flow optimization using positive programming," 18th IFAC World Congress, 2011.

[112] S. Sojoudi and S. H. Low, "Optimal charging of plug-in hybrid electric vehicles in smart grids," IEEE Power and Energy Society General Meeting, 2011.

[113] M. Kraning, E. Chu, J. Lavaei, and S. Boyd, "Dynamic network energy management via proximal message passing," Foundations and Trends in Optimization, vol. 1, no. 2, pp. 1-54, 2013.

[114] S. Sojoudi and J. Lavaei, "Convexification of optimal power flow problem by means of phase shifters," IEEE SmartGridComm, 2013.

[115] R. Madani, M. Ashraphijuo, and J. Lavaei, "Promises of conic relaxation for contingency-constrained optimal power flow problem," Allerton, 2014. 